\title{
Luminescence des ions activateurs dans lec matériaux inorganiques et applications
}

\author{
G. Boulon \\ Laboratoire de Physico-Chimie des Matériaux Luminescents, Unité Associée au CNRS $\mathrm{n}^{\circ} 442$, Université
} Claude Bernard Lyon I, 43, Bd du 11 Novembre 1918, 69622 Villeurbanne, France

(Reçu le 20 mars 1986, accepté le 15 mai 1986)

\begin{abstract}
Résumé. - Nous présentons les mécanismes de base de l'absorption d'un rayonnement de pompage et de l'émission de fluorescence des matériaux inorganiques luminescents et nous donnons la liste des principaux composés utilisés par la technologie moderne. Après avoir montré les relations entre les recherches conduites pour le développement des deux types de matériaux (luminophores polycristallins et monocristaux lasers) nous insistons plus sur l'état de l'art et les perspectives des recherches des luminophores pour l'éclairage, la télévision, les écrans renforçateurs de rayons $\mathrm{X}$ et les écrans électroluminescents.
\end{abstract}

\begin{abstract}
We present the basic mechanisms of the absorption of the pumping radiation and the fluorescence emission of inorganic luminescent materials and we give the list of main compounds used by modern technology. Then, we show there were connections between polycrystalline phosphors and laser-material research. We insist on the present status and the future development of phosphors for fluorescent lighting, TV screens, X-ray intensifier screens and electroluminescent devices.
\end{abstract}

\section{Introduction.}

Les matériaux luminescents font aujourd'hui, partie intégrante de notre environnement journalier. On les trouve presque partout pour leurs applications à l'éclairage dans les tubes fluorescents ou à la visualisation des écrans de télévision, à celle des écrans des nouveaux calculateurs ainsi que des écrans des machines à traitement de texte ou bien encore en France au Minitel. Il n'existera bientôt plus une maison qui ne les utilise pas sous leur forme polycristalline que l'on appelle soit des luminophores, soit des phosphores. La technologie moderne met aussi à profit ces composés luminescents dans les branches de pointes comme l'électronique avec les écrans d'oscilloscopes, les nouveaux écrans plats électroluminescents ou les écrans renforçateurs d'images de rayons $\mathrm{X}$, comme aussi tout ce qui touche à l'avionique. Sous leur forme monocristalline ou vitreuse, ces matériaux connaissent un essor remarquable pour leur utilisation croissante soit comme milieux lasers à solide, soit comme scintillateurs dans des domaines aussi variés que la physique nucléaire de haute énergie, la tomographie avec le développment des scanners, ou dans un avenir proche, comme concentrateurs solaires (Tableau I).

Dans la première partie de cet article nous présenterons les principaux matériaux utilisés ou en voie d'utilisation puis nous décrirons les principes de base qui permettent de comprendre les mécanismes d'émission de fluorescence. En fait, les matériaux inorganiques stables les plus connus sont les luminophores pour l'éclairage et la télévision et les lasers à solide. Il nous a paru intéressant de montrer quelles ont été les relations entre les recherches conduites pour chacun d'entre eux. La deuxième partie est plutôt réservée au développement des luminophores et des monocristaux sans s'attarder sur les lasers à solide qui font l'objet d'une autre publication de ce colloque.

\section{Principes de base liés à la luminescence.}

La figure 1 rappelle les principaux processus de l'interaction entre un ravonnement excitateur et la matière : pompage, absorption, émission spontanée de fluorescence et émission stimulée lorsque le milieu devient amplificateur dans un laser à solide. Alors que l'émission stimulée ou induite ne peut apparaître que dans la direction du faisceau de pompage ou de la direction imposée par la configuration géométrique des miroirs de la cavité oscillatrice du laser, l'émission spontanée est émise dans toutes les directions sous la forme de trains d'onde sans relation ni d'amplitude, ni de phase entre eux. Cette émission de trains d'onde incohérents de la fluorescence contraste avec la cohérence bien connue des trains d'onde de l'émission laser. En pratique, la 


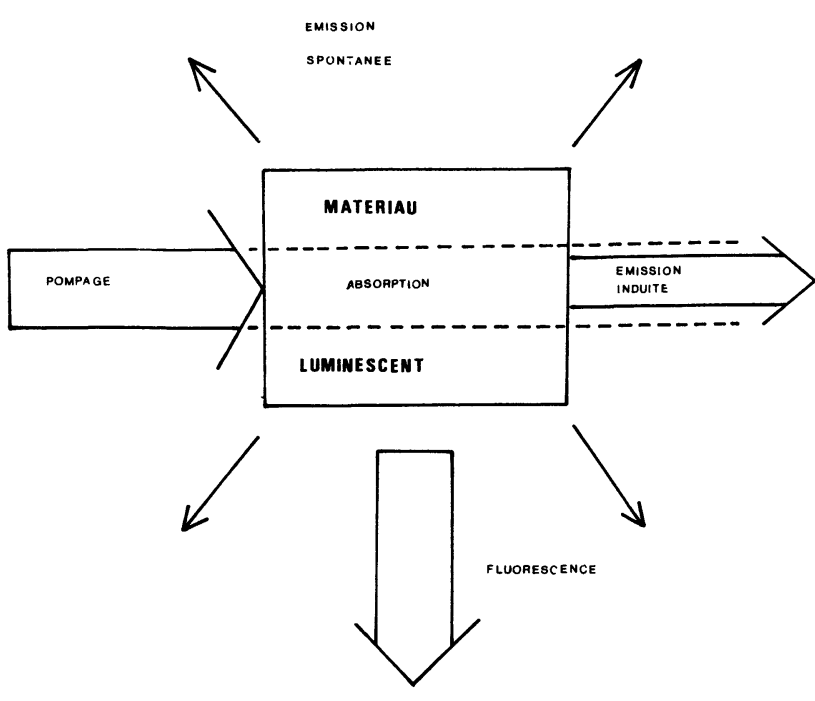

Fig. 1. - Représentation schématique de l'interaction entre le rayonnement excitateur (pompage) et le matériau luminescent : absorption du faisceau excitateur, émission spontanée de fluorescence et émission induite ou stimulée.

[Schematic representation of the interaction between the pumping beam and the luminescent solid-state material : absorption, spontaneous emission of fluorescence and stimulated (or induced) emission.]

production d'émission stimulée du laser nécessite généralement des monocristaux ou des verres dont la longueur des barreaux dépasse plusieurs millimètres puisque le coefficient d'amplification dépend de la longueur par une exponentielle. Si bien que les luminophores polycristallins dont les dimensions des grains sont de l'ordre du micron n'émettent de façon appréciable que l'émission spontanée. Selon le mode d'excitation, c'est-à-dire selon la nature du pompage, on rencontre le vocabulaire suivant :

- Le pompage optique par un rayonnement infra-rouge, visible ou ultraviolet induit la photoluminescence. Elle est mise en jeu dans les tubes fluorescents sous l'excitation ultraviolette des raies $\mathrm{du}$ mercure, dans les ballons fluorescents où le luminophore est additionné pour corriger la couleur de la lampe à vapeur de mercure, dans les lasers pompés par les lampes au xénon ou au krypton et dans les concentrateurs solaires soumis au flux du soleil.

- Le pompage par un champ électrique dû à l'application d'une tension supérieure à $100 \mathrm{~V}$ induit l'électroluminescence qui est à la base du fonctionnement des écrans plats des futurs appareils de télévision. Dans ce cas, il y a transport de charge dans le solide.

- Le pompage par un faisceau d'électrons accélérés sous vide induit la cathodoluminescence mise à profit pour les écrans de télévision et, maintenant, de calculateurs.

- Le pompage par les rayons $\mathrm{X}$ induit des émissions de fluorescence visible qui sont largement développées dans les écrans intensificateurs d'images pour la radiographie.
- Le pompage par les rayons $\gamma$ émis par les processus nucléaires peut être détecté par les cristaux scintillateurs aussi bien lors d'expériences de physique fondamentale que dans la détection des signaux en tomographie.

Ainsi un matériau luminescent est avant tout, soit un générateur, soit un convertisseur de lumière. On se doute que les mécanismes d'absorption et d'émission diffèrent d'une excitation à l'autre ce qui rend d'ailleurs assez difficile les transferts de connaissance entre les divers champs d'applications. On peut seulement affirmer que c'est la photoluminescence qui reste le point commun de cette classification puisqu'en fin de compte ce sont presque toujours les premiers niveaux d'énergie excités qui émettent la fluorescence visible que l'on cherche à exploiter. En conséquence, toute analyse spectrale de composés destinés aux applications de la luminescence devrait obligatoirement débuter par l'étude de la photoluminescence. Aujourd'hui, cette recherche bénéficie de l'emploi des lasers continus et à impulsions, d'excellente résolution et accordable entre $210 \mathrm{~nm}$ et $800 \mathrm{~nm}$.

On vient d'évoquer les niveaux d'énergie excités de l'ion activateur de la matrice hôte qui sont en réalité leur carte d'identité et on ne pourra plus s'en passer pour décrire précisément la luminescence. Les différents types de pompage cités précédemment vont, selon la valeur de leur énergie et aussi selon leur intensité, hisser un nombre plus ou moins grand d'électrons du niveau fondamental de l'ion activateur vers les niveaux excités proches (photoluminescence) ou très élevés (cathodoluminescence, rayons $\mathrm{X}$ et $\gamma$ ). La quantification de l'énergie a été aussi introduite pour les réseaux cristallins et les vibrations des ions qui le constituent. Une représentation schématique des positions relatives de l'ensemble des niveaux mis en jeux est montrée sur la figure 2 tandis que la figure 3 rappelle les éléments essentiels qui agissent sur l'obtention de la fluorescence :

Ce sont :

- La nature du réseau cristallin et la largeur de sa bande interdite entre la bande de valence et la bande de conduction.

- Les familles d'ions activateurs fluorescents : ions de transitions, ions terre rare, ions isoélectroniques du mercure, actinides indiqués sur le tableau II et, aussi, les groupements moléculaires propres aux réseaux comme les tungstates, molybdates, niobates, tantalates, sans oublier les centres colorés.

- L'énergie et l'intensité du pompage.

- La valeur de la température du bain thermique à laquelle est soumis le matériau luminescent.

On notera que les niveaux de l'ion activateur seront d'autant plus localisés que la bande interdite est large mais qu'ils peuvent recouvrir les bandes de valence et de conduction introduisant des phénomènes de délocalisation des électrons excités associés, dans un grand nombre de cas à des pertes de rendement lumineux. A cet égard, le succès rencontré par le rubis est dû, entre autres raisons, à la localisation des niveaux d'énergie du chrome $\mathrm{Cr}^{3+}$ 


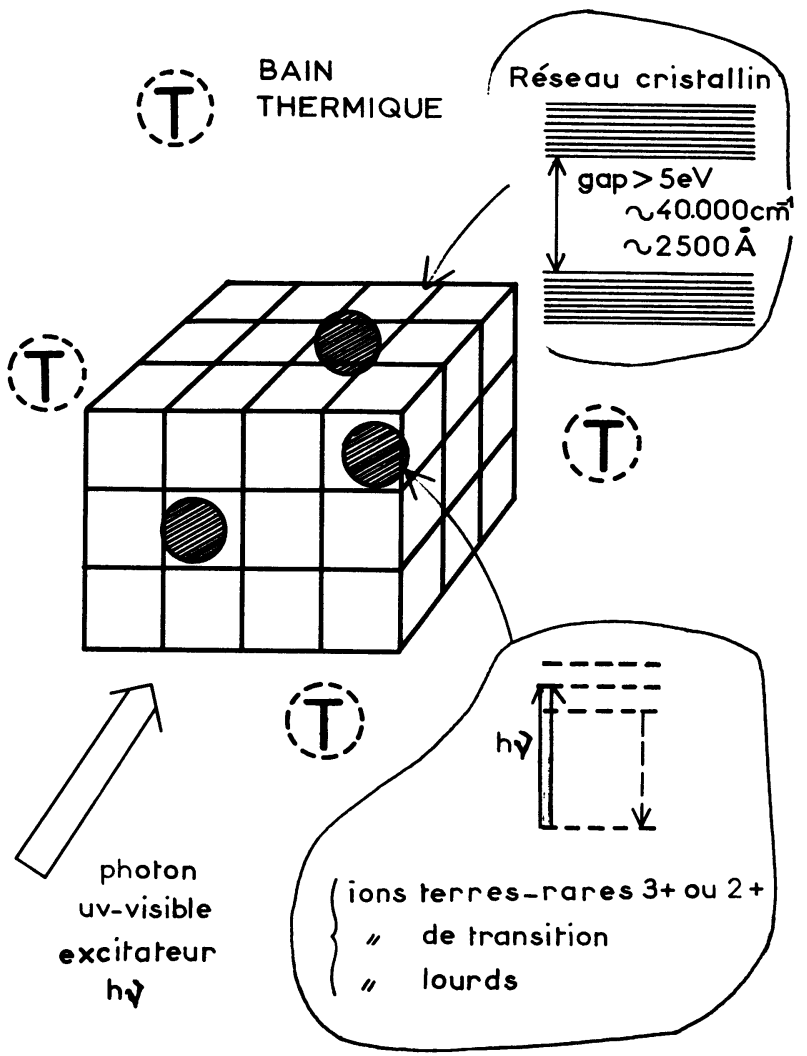

Fig. 2. - Interaction des niveaux d'énergie électroniques des ions constituant le réseau cristallin, des ions dopants et des vibrations des ions du réseau. Les ordres de grandeur approximatifs sont indiqués en $\mathrm{cm}^{-1}$.

[Electronic energy levels both of i) the internal ions inside the crystalline host ; ii) and the activator ions ; and the frequency spectrum of the vibrational modes. The approximate magnitude orders are noted in wavenumber $\left.\left(\mathrm{cm}^{-1}\right).\right]$

dans la bande interdite de l'alumine $(\sim 7 \mathrm{eV})$ comme le montre la figure 4 .

Lors de l'échange entre le rayonnement excitateur et les électrons des ions activateurs, un photon cède son énergie à un électron, lequel, après des relaxations non radiatives, contribue à l'émission du photon de fluorescence. La matrice luminescente constitue donc un réservoir d'électrons actifs pour le processus de luminescence que l'on définit par la population du niveau fondamental. Cette population, exprimée en nombre d'atomes par $\mathrm{cm}^{3}\left(\sim 10^{19}\right.$ à $10^{22}$ ) est directement reliée à la teneur des ions activateurs.

Nous ne reviendrons pas sur la recherche des niveaux d'énergie des ions et sur leurs notations spectroscopiques en fonction des valeurs, à la fois, des énergies de configuration sur les couches électroniques, de la répulsion interélectronique et du couplage spin-orbite. Nous voulons simplement mentionner la grosse influence de la symétrie ponctuelle du site occupé sur la décomposition des niveaux d'énergie en sous-niveaux Stark due au champ électrique cristallin résultant qui dépend de la dis-

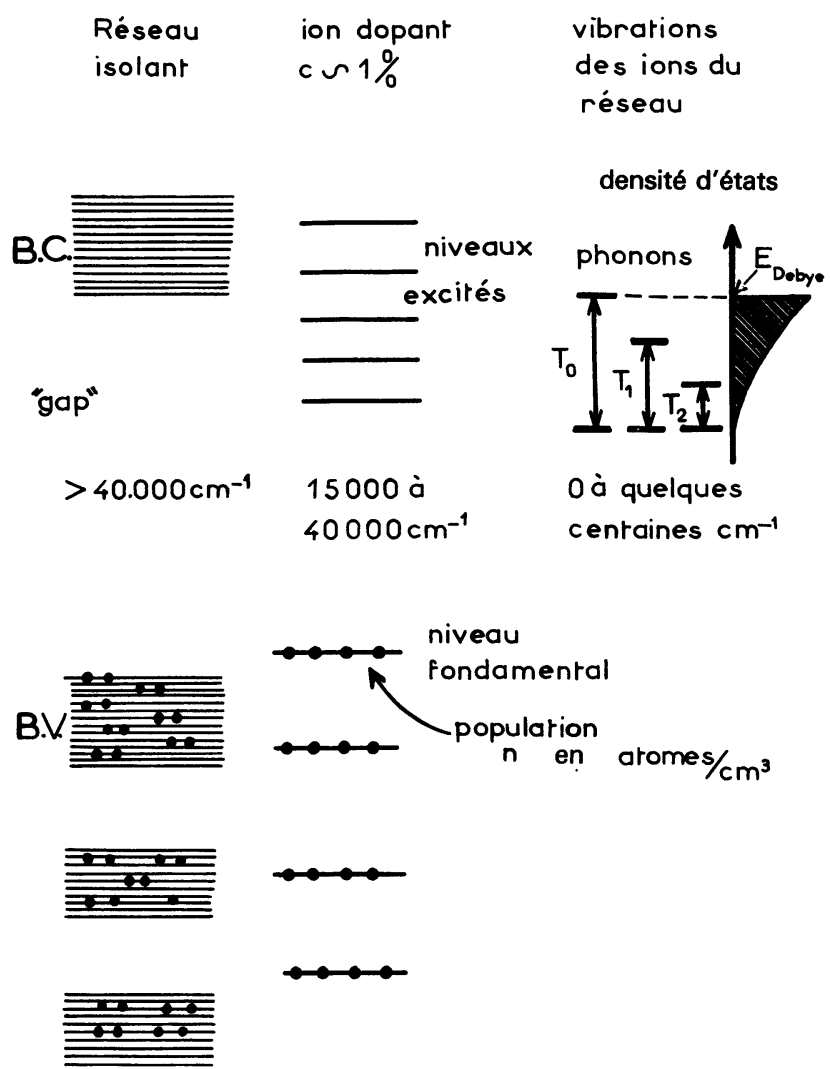

Fig. 3. - Principaux éléments impliqués pour l'obtention de l'émission de fluorescence d'un matériau cristallin contenant des ions activateurs.

[Main elements connected with the production of the emission of fluorescence by activator ion doped hosts.]

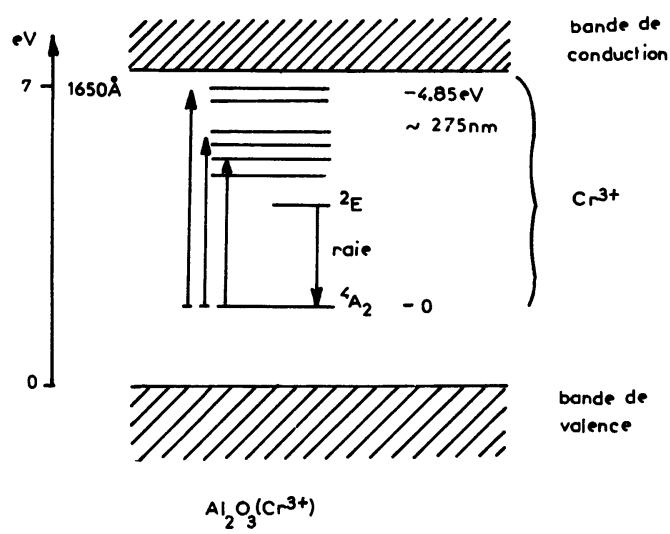

Fig. 4. - Exemple du rubis : localisation des niveaux d'énergie de l'ion chrome $\mathrm{Cr}^{3}$ dans la bande interdite de l'alumine $\mathrm{Al}_{2} \mathrm{O}_{3}$.

[Case of the ruby solid state laser localization of the $\mathrm{Cr}^{3+}$ energy levels in the $\mathrm{Al}_{2} \mathrm{O}_{3}$ forbidden gap.]

tance $r$ des ions les plus proches voisins, donc de la coordinence et de la distribution des charges (Fig. 5).

Cependant la description précédente n'est pas suffisante. Il convient de l'affiner en tenant compte 
Tableau I. - Applications des polycristaux luminescents.

Tubes fluorescents trichromatiques

$$
\mathrm{BaMg}_{2} \mathrm{Al}_{16} \mathrm{O}_{27}\left(\mathrm{Eu}^{2+}\right), \mathrm{CeMgAl}_{11} \mathrm{O}_{19}\left(\mathrm{~Tb}^{3+}\right), \mathrm{Y}_{2} \mathrm{O}_{3}\left(\mathrm{Eu}^{3+}, \quad \mathrm{CeGdMg} \mathrm{B} \mathrm{O}_{10}\left(\mathrm{~Tb}^{3+}\right)\right.
$$

Lampes compactes nouvelle génération

$$
3 \mathrm{Sr}_{3}\left(\mathrm{PO}_{4}\right)_{2}-\mathrm{CaCl}_{2}\left(\mathrm{Eu}^{2+}\right), \quad \mathrm{LaPO}_{4}\left(\mathrm{Ce}^{3+}, \mathrm{Tb}^{3+}\right), \quad \mathrm{Y}_{2} \mathrm{O}_{3}\left(\mathrm{Eu}^{3-}\right) .
$$

Ecrans TV

$$
\mathrm{ZnS}\left(\mathrm{Ag}^{+}, \mathrm{Al}^{3+}\right), \quad \mathrm{ZnS}\left(\mathrm{Cu}^{+}, \mathrm{Al}^{3+}\right), \quad \mathrm{Y}_{2} \mathrm{O}_{2} \mathrm{~S}\left(\mathrm{Eu}^{3+}\right)
$$

Ecrans haute résolution

$$
\mathrm{Y}_{3} \mathrm{Al}_{5} \mathrm{O}_{12}\left(\mathrm{Ce}^{3+}\right)
$$

Ecrans renforçateurs de rayons $\mathrm{X}$

$$
\mathrm{CaWO}_{4} \quad \mathrm{Gd}_{2} \mathrm{O}_{2} \mathrm{~S}\left(\mathrm{~Tb}^{3+}\right)
$$

Couches électroluminescentes

$$
\mathrm{SrS}\left(\mathrm{Ce}^{3+}\right) \quad \mathrm{ZnS}-\mathrm{TbF}_{3} \quad \mathrm{ZnS}-\mathrm{SmF}_{3} \quad \mathrm{ZnS}\left(\mathrm{Mn}^{2+}\right)
$$

Tableau I (suite). - Applications des cristaux et verres luminescents.

Scintillateurs

$$
\mathrm{NaI}\left(\mathrm{Tl}^{+}\right), \quad \mathrm{Bi}_{4} \mathrm{Ge}_{3} \mathrm{O}_{12}, \quad \mathrm{Y}_{3} \mathrm{Al}_{5} \mathrm{O}_{12}\left(\mathrm{Ce}^{3+}\right), \quad \text { verres }\left(\mathrm{Ce}^{3+}\right)
$$

Concentrateurs solaires

$$
\text { verres }\left(\mathrm{Nd}^{3+}, \mathrm{Yb}^{3+}\right), \quad \text { verres }\left(\mathrm{Cr}^{3+}, \mathrm{Nd}^{3+}\right), \quad \text { vitrocéramiques }\left(\mathrm{Cr}^{3+}, \mathrm{Nd}^{3+}\right)
$$

Matériaux lasers

$$
\begin{aligned}
& \mathrm{Al}_{2} \mathrm{O}_{3}\left(\mathrm{Cr}^{3+}\right), \quad \mathrm{Y}_{3} \mathrm{Al}_{5} \mathrm{O}_{12}\left(\mathrm{Nd}^{3+}\right), \quad \mathrm{LiYF}_{4}\left(\mathrm{Nd}^{3+}\right), \quad \mathrm{LaMgAl}_{11} \mathrm{O}_{19}\left(\mathrm{Nd}^{3+}\right), \\
& \mathrm{Gd}_{3} \mathrm{Sc}_{2} \mathrm{Ga}_{3} \mathrm{O}_{12}\left(\mathrm{Nd}^{3+}\right) \text { ei }\left(\mathrm{Cr}^{3+}-\mathrm{Nd}^{3+}\right), \quad \mathrm{BeAl}_{2} \mathrm{O}_{4}\left(\mathrm{Cr}^{3+}\right), \quad \mathrm{BeAl}_{2}\left(\mathrm{SiO}_{3}\right)_{6}\left(\mathrm{Cr}^{3+}\right) \\
& \mathrm{Ca}_{3} \mathrm{Al}_{2} \mathrm{Ge}_{3} \mathrm{O}_{12}\left(\mathrm{Cr}^{3+}\right), \quad \mathrm{Al}_{2} \mathrm{O}_{3}\left(\mathrm{Ti}^{3+}\right) \text {, } \\
& \mathrm{LiYF}_{4}\left(\mathrm{Ce}^{3+}\right), \quad \mathrm{LaF}_{3}\left(\mathrm{Ce}^{3+}\right), \quad \mathrm{MgF}_{2}\left(\mathrm{Ni}^{2+}\right) \text { et }\left(\mathrm{Co}^{2+}\right), \quad \mathrm{KMgF}_{3}\left(\mathrm{~V}^{2+}\right), \quad \text { verres }\left(\mathrm{Nd}^{3+}\right)
\end{aligned}
$$

réellement des vibrations des ions les plus proches voisins du cation central. Une bonne approximation est celle des oscillateurs harmoniques traduits sur la figure 6 par des niveaux vibrationnels associés à chaque niveau électronique mais aussi par des courbes d'énergie potentielle, paraboliques, appelées courbes de configuration, lorsqu'on représente la variation de l'énergie en fonction d'une distance arbitraire $r$. A chaque niveau vibrationnel correspond une fonction d'onde $\psi$ dont l'amplitude est maximale pour la valeur de $r$ égale à l'abscisse de la parabole. La figure 6 montre, par exemple, les fonctions d'onde représentatives de $v=0$ du niveau fondamental ${ }^{7} \mathrm{~F}_{0}$ de l'ion $\mathrm{Eu}^{3+}$ et de $v^{\prime}=0$ et $v^{\prime}=2$ $\mathrm{du}$ premier niveau excité ${ }^{5} \mathrm{D}_{0}$. La transition ${ }^{7} \mathrm{~F}_{0}(v=0) \rightarrow{ }^{5} \mathrm{D}_{0}(v=0)$ aux très basses températures est appelée transition à 0 phonon. Elle est localisée vers $580 \mathrm{~nm}$ dans les solides, c'est-à-dire, dans un domaine spectral facile à atteindre et n'est d'ailleurs pas décomposée par le champ cristallin (passage de $J=0$ à $J=0$ avec les notations classi- ques des termes spectroscopiques) ce qui fait tout son intérêt pour sonder les sites occupés par l'europium dans les matrices cristallines.

L'exemple choisi de la terre rare $\mathrm{Eu}^{3+}$ pourrait être étendu à toutes les terres rares de configuration électronique $4 \mathrm{f}^{n}$ qui est protégée de l'environnement extérieur par les couches périphériques $5 \mathrm{~s}^{2}$ et $5 \mathrm{p}^{6}$, les trois électrons ayant été prélevés sur les couches $5 \mathrm{~d}$ et $6 s^{2}$. A cause de cette protection, la parabole du niveau excité n'est pas décalée par rapport à celle du niveau fondamental. Il en résulte que les raies d'absorption (ou d'excitation) et d'émission de fluorescence ${ }^{5} \mathrm{D}_{0} \rightleftarrows{ }^{7} \mathrm{~F}_{0}$ sont confondues. On dit qu'il n'y a pas de décalage de Stokes entre l'absorption et l'émission. Cependant, ces décalages de Stokes existent pour un grand nombre de configurations des familles d'activateurs. Sur la figure 7 , on observe alors que le recouvrement spectral n'est total que pour la raie à 0 phonon et que les spectres d'absorption et de fluorescence comprennent une partie vibronique souvent dominante. On caractérise ce 


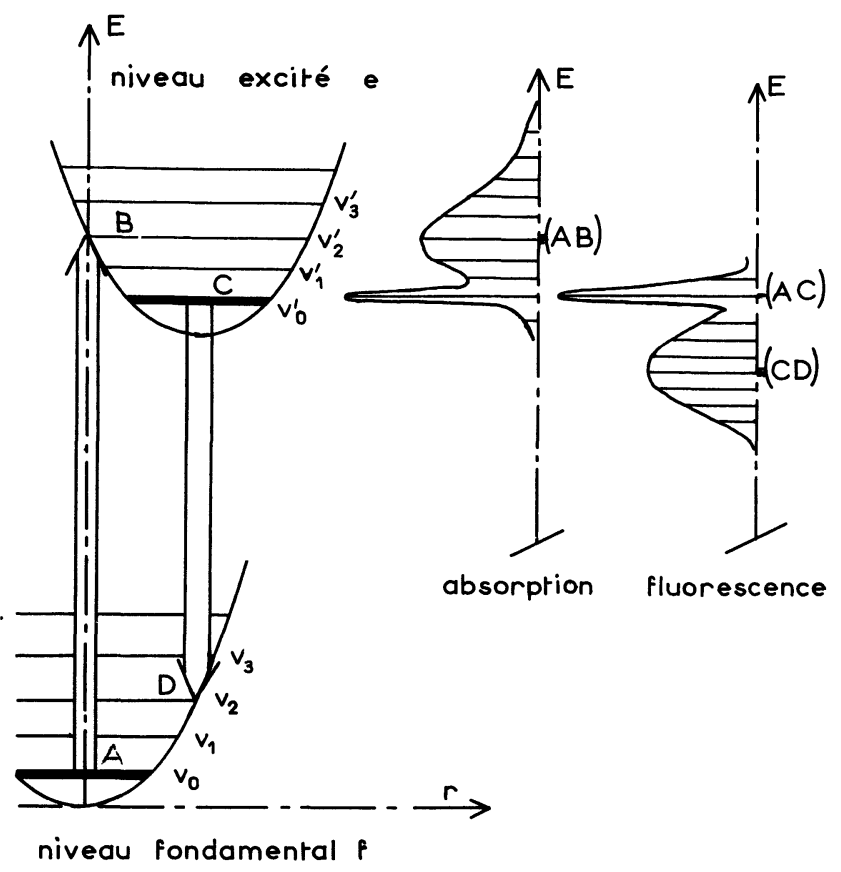

Fig. 7. - Courbes de configuration relative à une transition entre un niveau fondamental et un niveau excité soumis à un fort couplage électron-phonon. Les spectres vibroniques sont alors décalés vers les grandes énergies pour l'absorption et vers les faibles énergies pour l'émission ; ils correspondent aux transitions entre les niveaux vibrationnels $\mathrm{v}$ et $\mathrm{v}^{\prime}$. La transition à 0 phonon est la transition $\mathrm{AC}$ commune aux spectres d'absorption (dont le maximum est $\mathrm{AB}$ ) et d'émission (dont le maximum est CD).

[Configurational coordinate curves of a transition associated with a strong electron-phonon coupling. The vibronic spectra are shifted to higher energies for the absorption and to lower energies for the emission; there are the transitions between the vibrational levels $\mathrm{v}$ and $\mathrm{v}^{\prime}$. AC is the 0-phonon transition which is commun to absorption (which the maximum is $\mathrm{AB}$ ) and to emission (which the maximum is $\mathrm{CD})$.]

des atomes du réseau c'est-à-dire sur l'interaction électron-phonon. Chaque transition est caractérisée par une largeur homogène qui dépend de deux facteurs : la température par cette interaction et la durée de vie radiative du niveau excité $\tau$ liée à la largeur naturelle $\Delta \nu$ par la relation d'incertitude $\tau . \Delta \nu \sim \frac{1}{2 \pi}$. Pour des raisons expérimentales il est souvent difficile d'atteindre la largeur homogène en dessous de $50 \mathrm{~K}$. Au-dessus de cette température la variation thermique des largeurs homogènes renseigne sur les mécanismes d'interaction électron-phonon. Si on ne dispose pas de lasers accordables en fréquences et de bonne résolution spectrale, les largeurs obtenues traduisent plutôt les largeurs inhomogènes, enveloppe des raies homogènes des multisites d'ions perturbés par des impuretés ou contraintes locales qu'il est d'ailleurs bien difficile d'éliminer lors de la croissance. On comprend ainsi tout l'intérêt de la technique de spectroscopie laser dite de rétrécissement de la raie de fluorescence illustrée sur la figure 8 .

L'influence de la température sur le rendement de l'émission de fluorescence peut être comprise à partir des courbes de configuration dessinées sur la figure 9a. On voit, en effet, que les paraboles du niveau fondamental et du niveau excité se coupent en $T$ et que si l'on franchit la barrière de potentiel définie par $W$, appelée énergie d'activation thermique, les transitions s'effectuent sans émission de photon, le système retournant au niveau fondamental à partir des niveaux vibrationnels élevés libérant son énergie sous forme de phonons au réseau. La probabilité de transitions non radiatives augmente donc avec l'élévation de la température via le peuplement des niveaux vibrationnels. Il peut même exister des matériaux qui absorbent l'énergie excitatrice mais n'émettent pas lorsque les deux paraboles sont trop décalées (Fig. 9b).

La connaissance de ce paramètre $W$ est importante pour prévoir le comportement thermique du matériau luminescent. Si l'on s'en tient au modèle développé ici avec seulement deux niveaux il faut s'attendre à ne pas enregistrer d'extinction thermique avec les ions dont les décalages de Stokes sont nuls. Cette tendance semble vérifiée pour les terres rares trivalentes et pour l'ion $\mathrm{Cr}^{3+}$ dans $\mathrm{Al}_{2} \mathrm{O}_{3}$.

Tableau III. - Les principaux ions activateurs dans les solides.

\begin{tabular}{|c|c|c|c|c|}
\hline $\begin{array}{c}\text { Nature de la } \\
\text { transition }\end{array}$ & $\mathrm{f}-\mathrm{f}$ & $\mathrm{f}-\mathrm{d}$ & $\mathrm{d}-\mathrm{d}$ & $\mathrm{s}-\mathrm{p}$ \\
\hline Activateurs & terres rares $3^{+}$ & terres rares $3^{+}$ & ions de transitions & $\begin{array}{c}\text { ions lourds } \\
\text { centres colorés }\end{array}$ \\
\hline $\begin{array}{c}\text { Intensité du } \\
\text { couplage }\end{array}$ & faible & intermédiaire & $\begin{array}{c}\text { intermédiaire } \\
+ \text { fort }\end{array}$ & 10 à 100 \\
\hline$S_{0}$ & $10^{-2}$ à $10^{-1}$ & 1 à 6 & 1,5 à 10 & \\
\hline
\end{tabular}




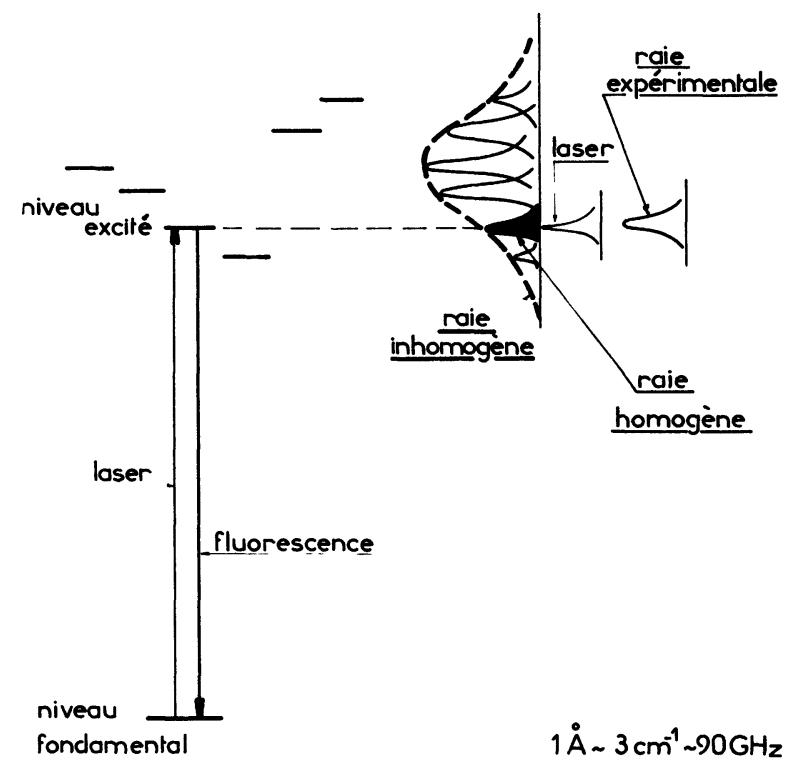

Fig. 8. - Illustration de la technique de spectroscopie laser par rétrécissement de la raie de fluorescence sur la raie inhomogène d'une transition à 0 -phonon d'un ion activateur. La correspondance indiquée entre les grandeurs spectrales n'est valable qu'autour de $\lambda=500 \mathrm{~nm}$ (5000 $\AA$ ).

[Laser-spectroscopy techniques : fluorescence line narrowing applied on the inhomogeneous line in conjunction with the 0-phonon transition. The correspondence between the spectral parameters are calculated to be around $\lambda=500 \mathrm{~nm}(5000 \AA)$.]

Cependant, il existe d'autres niveaux supérieurs qui subissent un couplage élevé avec le réseau qui contribuent largement aux désexcitations non radiatives. C'est le cas des bandes de transfert de charge $\mathrm{Eu}^{3+}-\mathrm{O}^{2-}$ représentées sur la figure 10 correspondant au passage d'un électron de l'oxygène sur l'europium.

Ces relaxations non radiatives entre les paraboles des niveaux excités expliquent les profils de raies ou de bandes des ions terre rare et des ions de transitions. Nous avons résumé sur les figures 11 et 12 l'influence du champ cristallin sur l'ion $\mathrm{Eu}^{2+}$ (raie de la configuration $4 \mathrm{f}^{7}$ et bande large de la configuration $4 \mathrm{f}^{6}-5 \mathrm{~d}$ ) et sur l'ion $\mathrm{Cr}^{3+}$ (raie ${ }^{2} \mathrm{E} \rightarrow{ }^{4} \mathrm{~A}_{2}$ et bande ${ }^{4} \mathrm{~T}_{2} \rightarrow{ }^{4} \mathrm{~A}_{2}$ ) pour lequel l'équilibre thermique qui intervient dans le cas d'un champ intermédiaire donne lieu à un spectre qui est la superposition des spectres à champ fort et à champ faible.

Enfin, nous avons schématisé sur les figures 13, 14 et 15 les transitions des terres rares utilisées dans les luminophores et les lasers à solide et celles des ions de transition dont les bandes larges sont l'objet de recherches pour les lasers à solide accordables dans le proche infrarouge [1-3].

\section{Probabilités d'absorption, d'émission spontanée et d'émission induite d'une transition.}

Tous les bilans de populations des niveaux d'un ion activateur nécessitent la connaissance des probabilités d'absorption, d'émission spontanée et induite appelées aussi coefficients d'Einstein. Si $n_{1}$ désigne la population du niveau fondamental (1) et $n_{2}$ celle du niveau excité (2) on définit les différents mécanismes de la manière suivante :
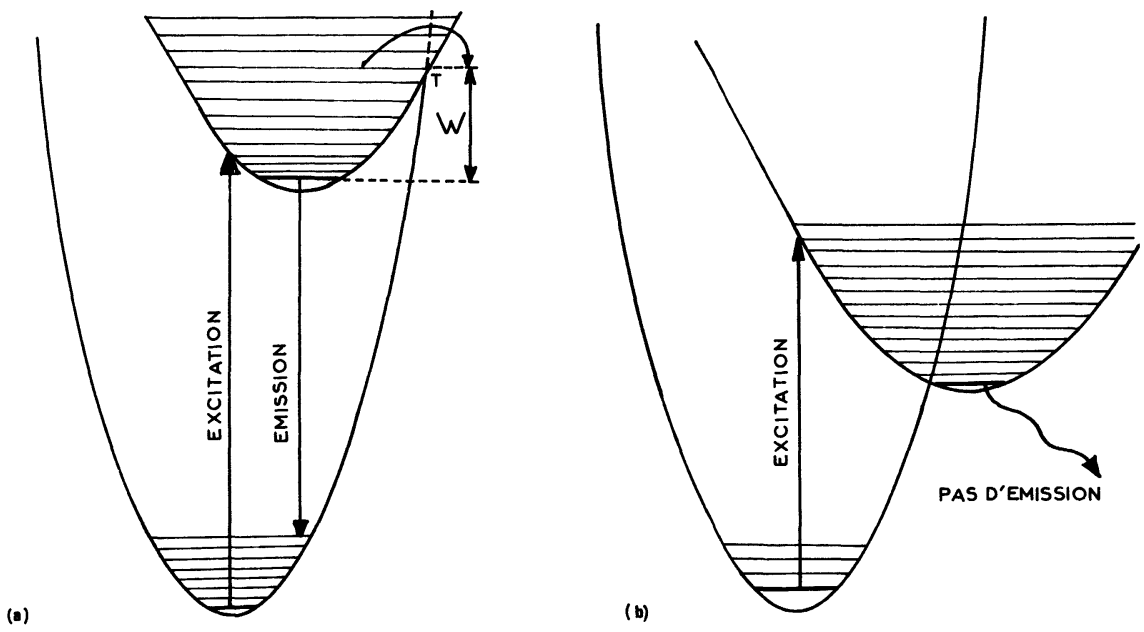

Fig. 9 - Modélisation de l'influence de la température sur les processus d'extinction de la fluorescence d'un centre activateur. a) Barrière ou énergie thermique $W$ qu'il faut surmonter pour dissiper l'énergie sous forme non radiative. b) La barrière n'existe pas et toute l'énergie absorbée est dissipée sous forme non radiative.

[Model for temperature influence on the quenching of fluorescence. a) thermal activation energy $W$ to surpass in order to produce non-radiative energy transitions. b) There is no potential barrier and all absorbed energy is dissiped as nonradiative transitions.] 


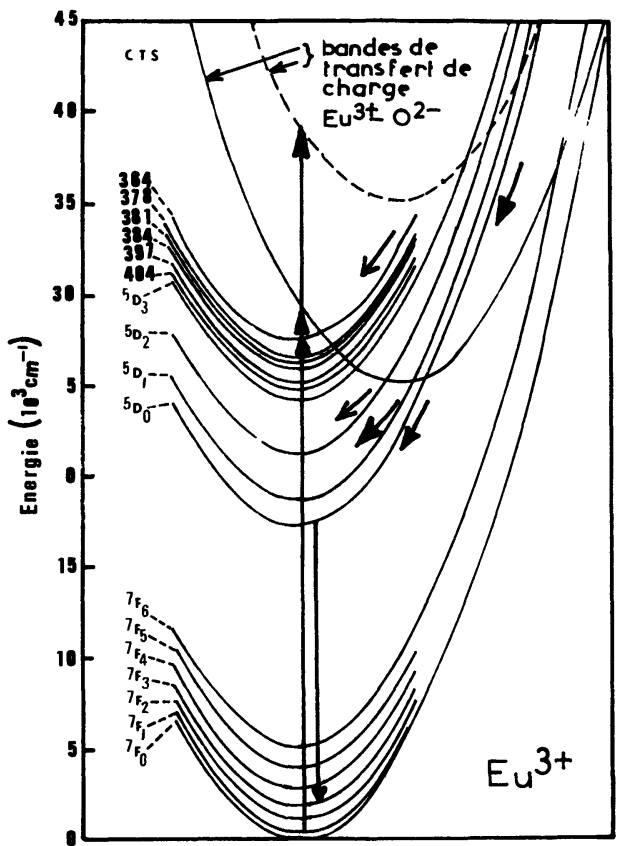

Fig. 10 - Courbes de configuration des états de l'ion $\mathrm{Eu}^{3+}$ dans les solides. Les paraboles des états de transfert de charge $\mathrm{Eu}^{3+}-\mathrm{O}^{2-}$ qui servent à l'excitation principale de l'ion luminescent en raison de leur coefficient d'absorption élevé subissent un décalage de Stokes important et jouent un rôle de pont intermédiaire entre états de la configuration $4 f^{6}$. Ils contribuent donc à la production d'émission non radiative par le haut des paraboles des états du niveau fondamental. Les flèches symbolisent l'alimentation des niveaux excités les plus bas.

[Configurational coordinate curves associated with $\mathrm{Eu}^{3+}$ ion transitions in solid-state hosts. The $\mathrm{Eu}^{3+}-\mathrm{O}^{2-}$ charge transfer states are used as an efficient pump. There is an important Stokes shift allowing intermediate bridges to $4 f^{6}$ states and then giving rise to non-radiative transitions via the highest vibrational levels of the ground state parabola. The arrows indicate the way to populate the lowest excited levels.] $A_{21}$

- Coefficient d'Einstein d'émission spontanée

$$
\frac{\mathrm{d} n_{2}}{\mathrm{~d} t}=-A_{21} n_{2} \text { et } n_{2}=n_{2}(0) \mathrm{e}^{-t / \tau}
$$

avec

$$
A_{21}=\frac{1}{\tau}
$$

$\tau$ désigne la durée de vie moyenne du niveau excité qui varie de la nanoseconde à plusieurs millisecondes :

$$
\begin{array}{ll}
\tau\left({ }^{2} \mathrm{E} \text { de } \mathrm{Cr}^{3+} \text { dans } \mathrm{Al}_{2} \mathrm{O}_{3}\right) & : 3 \mathrm{~ms} \\
\tau\left({ }^{4} \mathrm{~F}_{3 / 2} \text { de } \mathrm{Nd}^{3+} \text { dans YAG }\right) & : 240 \mu \mathrm{s} \\
\tau\left(5 \mathrm{~d} \text { de } \mathrm{Ce}^{3+} \text { dans YAG }\right) & : 80 \mathrm{~ns} .
\end{array}
$$

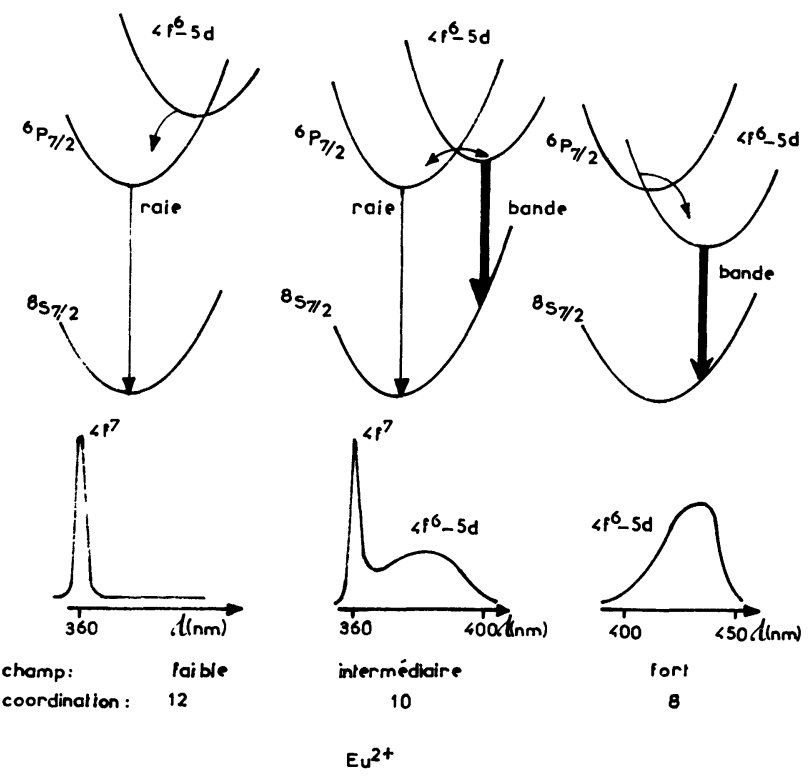

Fig. 11 - Mécanismes d'émission internes à l'ion $\mathrm{Eu}^{2+}$ selon l'intensité du champ cristallin après pompage sur les bandes larges ultraviolettes de la configuration $4 \mathrm{f}^{6}-5 \mathrm{~d}$ entre 200 et $400 \mathrm{~nm}$. La première transition ${ }^{8} \mathrm{~S}_{7 / 2} \rightarrow{ }^{7} \mathrm{P}_{7 / 2}$ de la configuration $4 \mathrm{f}^{7}$ localisée vers $360 \mathrm{~nm}$ ne dépend pratiquement pas du site occupé, par contre les bandes d'absorption de $4 f^{6}-5 d$ en sont très sensibles. La position des paraboles des niveaux de $4 \mathrm{f}^{6}-5 \mathrm{~d}$ explique l'évolution du spectre de fluorescence d'une raie (champ faible d'une coordination élevée) vers une bande (champ fort d'une coordination plus faible).

[Emission mechanisms in associated with $\mathrm{Eu}^{2}+$ ion depending on the crystalline field strength after pumping the uv broad bands of the $4 \mathrm{f}^{6}-5 \mathrm{~d}$ configuration between 200 and $400 \mathrm{~nm}$. The ${ }^{8} \mathrm{~S}_{7 / 2} \rightarrow{ }^{6} \mathrm{P}_{7 / 2}$ first transition of the $4 \mathrm{f}^{7}$ configuration is located around $360 \mathrm{~nm}$. It does not depend too much on the occupied site whereas the absorption bands of the $4 f^{6}-5 d$ configuration do. The location of the $4 f^{6}-5 d$ state parabola allows us to explain the spectral dependence from a line (weak crystal field with a high coordination) to a band (strong crystal field with a weaker coordination).]

- Coefficient d'Einstein d'absorption : $B_{12}$

$$
\frac{\mathrm{d} n_{1}}{\mathrm{~d} t}=-B_{12} \rho(\nu) n_{1}
$$

$\rho(\nu)$ est la densité spectrale énergétique de la source excitatrice. L'intensité transmise par un matériau d'épaisseur $x$ est

$$
I(x)=I(0) \mathrm{e}^{-\alpha x}
$$

avec

$$
\alpha(\nu)=\frac{\lambda^{2}}{8 \pi \tau}\left(n_{1}-n_{2}\right)
$$

lorsque les facteurs de dégénérescence sont égaux à l'unité pour (1) et (2); $\lambda$ désigne la longueur d'onde 

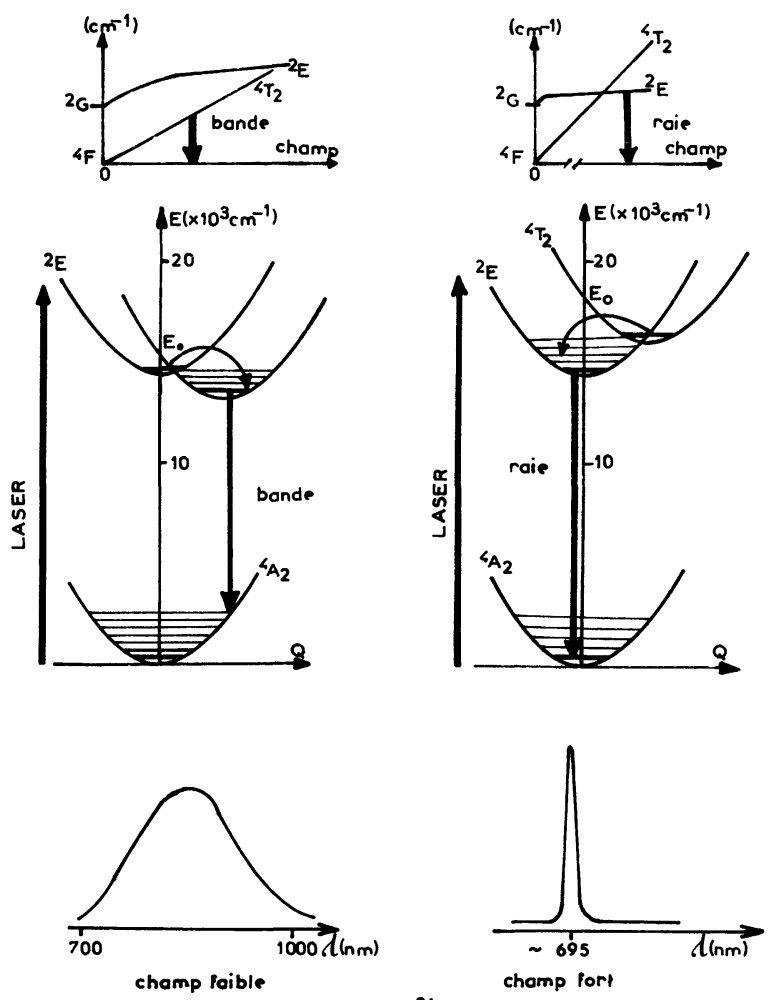

$\mathrm{Cr}^{3+}$

Fig. 12. - Mécanismes d'émission internes à l'ion $\mathrm{Cr}^{3+}$ selon l'intensité du champ cristallin décrit par les diagrammes d'énergie de Tanabe et Sugano après pompage sur les niveaux ${ }^{4} \mathrm{~T}_{2},{ }^{4} \mathrm{~T}_{1}$. Il y a compétition entre les transitions ${ }^{2} \mathrm{E} \rightarrow{ }^{4} \mathrm{~A}_{2}$ (raie fine) et ${ }^{4} \mathrm{~T}_{2} \rightarrow{ }^{4} \mathrm{~A}_{2}$ (bande large). Nous n'avons décrit que les deux cas extrêmes (champ fort et champ faible) mais il existe aussi le cas intermédiaire illustré par un spectre de superposition de la raie et de la bande.

[Emission mechanisms in association with $\mathrm{Cr}^{3+}$ ion depending on the crystalline field strength with is described by Tanabe and Sugano's energy diagram. The pumping acts on ${ }^{4} \mathrm{~T}_{2}$ and ${ }^{4} \mathrm{~T}_{1}$ levels. There is competition between the ${ }^{2} \mathrm{E} \rightarrow{ }^{4} \mathrm{~A}_{2}$ sharp line and the ${ }^{4} \mathrm{~T}_{2} \rightarrow{ }^{4} \mathrm{~A}_{2}$ broad band. We describe only the two extreme cases : strong and weak crystal field but there may appear an intermediate case with an overlap of the line and the band.]

de la transition et rappelons la relation entre $A_{21}$ et $B_{12}$ :

$$
\frac{A_{21}}{B_{12}}=\frac{8 \pi h}{\lambda^{3}} .
$$

On a pris l'habitude de définir ce coefficient d'absorption $\alpha(\nu)$ en $\mathrm{cm}^{-1}$ plutôt que $B_{12}$. Très souvent on introduit la notion de force d'oscillateur $f$ (sans dimension) pour caractériser la probabilité d'absorption d'une transition (1) $\rightarrow$ (2) :

$f \sim 0.1$ : transitions permises par le changement de parité entre configurations

$$
4 f^{n} \rightarrow 4 f^{n-1}-5 \mathrm{~d} \text { des terres rares }
$$

REVUE dE PHYSIQUE APPLIQUÉE. - T. 21, N 11, NOVEMBRE 1986 $n \mathrm{~s}^{2} \rightarrow n \mathrm{~s} n \mathrm{p}$ des ions isoélectroniques du mercure

$$
f \sim 10^{-2} \text { à } 10^{-4}
$$

$3 \mathrm{~d} \rightarrow 4 \mathrm{~s}$ et $4 \mathrm{~d} \rightarrow 5 \mathrm{~s}$ des ions de transitions

$f \sim 10^{-5}$ à $10^{-9}$ : transitions intraconfigurationnelles des terres rares $\left(4 f^{n}\right)$.

- Coefficient d'émission induite $B_{21}=B_{12}$

$$
\frac{\mathrm{d} n_{2}}{\mathrm{~d} t}=-B_{21} \rho(\nu) n_{2} \text {. }
$$

On rappelle que lorsque le milieu est devenu amplificateur :

$$
\begin{aligned}
& a(\nu)=-\alpha(\nu) \\
& a(\nu)>0 \text { si } n_{2}>n_{1}
\end{aligned}
$$

après l'inversion de population [4].

\section{Transfert d'énergie.}

Les transferts d'énergie entre 2 ou plusieurs ions du réseau cristallin jouent un rôle considérable sur l'ensemble des phénomènes de luminescence même lorsque le dopant a été introduit en petite quantité. Ils sont maintenant mis à profit dans de nombreux luminophores pour l'éclairage (transferts $\mathrm{Ce}^{3+} \rightarrow$ $\mathrm{Ce}^{3+} \rightarrow \mathrm{Gd}^{3+} \rightarrow \mathrm{Tb}^{3+} \rightarrow$ par exemple) et dans les lasers à solide (transfert $\mathrm{Cr}^{3+} \rightarrow \mathrm{Nd}^{3+}$ dans le grenat $\left.\mathrm{Gd}_{3} \mathrm{Sc}_{2} \mathrm{Ga}_{3} \mathrm{O}_{12}\right)$.

A titre indicatif il est facile de comprendre sur la figure 16 que le transfert entre l'ion sensibilisateur $S$ et l'ion activateur A est la conséquence du recouvrement spectral important entre le spectre d'absorption $g_{\mathrm{A}}(\nu)$ de A et celui d'émission $g_{\mathrm{S}}(\nu)$ de $\mathrm{S}$.

Les transferts peuvent être modélisés soit par un type résonnant radiatif, soit par un type résonnant non radiatif, soit, enfin être assistés par les phonons [5] :

- transfert résonnant radiatif : il y a absorption par A du photon émis par S. C'est un transfert à longue distance, sa probabilité est proportionnelle à $1 / R^{2}$. Il y a piégeage du photon dans le cristal. Expérimentalement, on observe une modification du profil du spectre d'émission de $\mathrm{S}$ en présence de A.

La probabilité est donnée par

$$
\begin{aligned}
& P_{\mathrm{SA}}=\frac{\sigma_{\mathrm{A}}}{4 \pi R^{2}} \cdot \frac{1}{\tau_{\mathrm{S}}} \cdot \int g_{\mathrm{S}}(\nu) \cdot g_{\mathrm{A}}(\nu) \mathrm{d} \nu \\
& \sigma_{\mathrm{A}}: \text { section efficace d'absorption de A. }
\end{aligned}
$$

- transfert résonnant non-radiatif : il y a interactions multipolaires dipôle-dipôle $(s=6)$, dipôlequadrupôle $(s=8)$, quadrupôle-quadrupôle $(s=10)$ ou interaction d'échange aux courtes distances.

La probabilité est donnée par

$$
P_{\mathrm{SA}}=\frac{1}{\tau_{\mathrm{S}}} \cdot\left\{\frac{R_{0}}{R}\right\}^{s}
$$



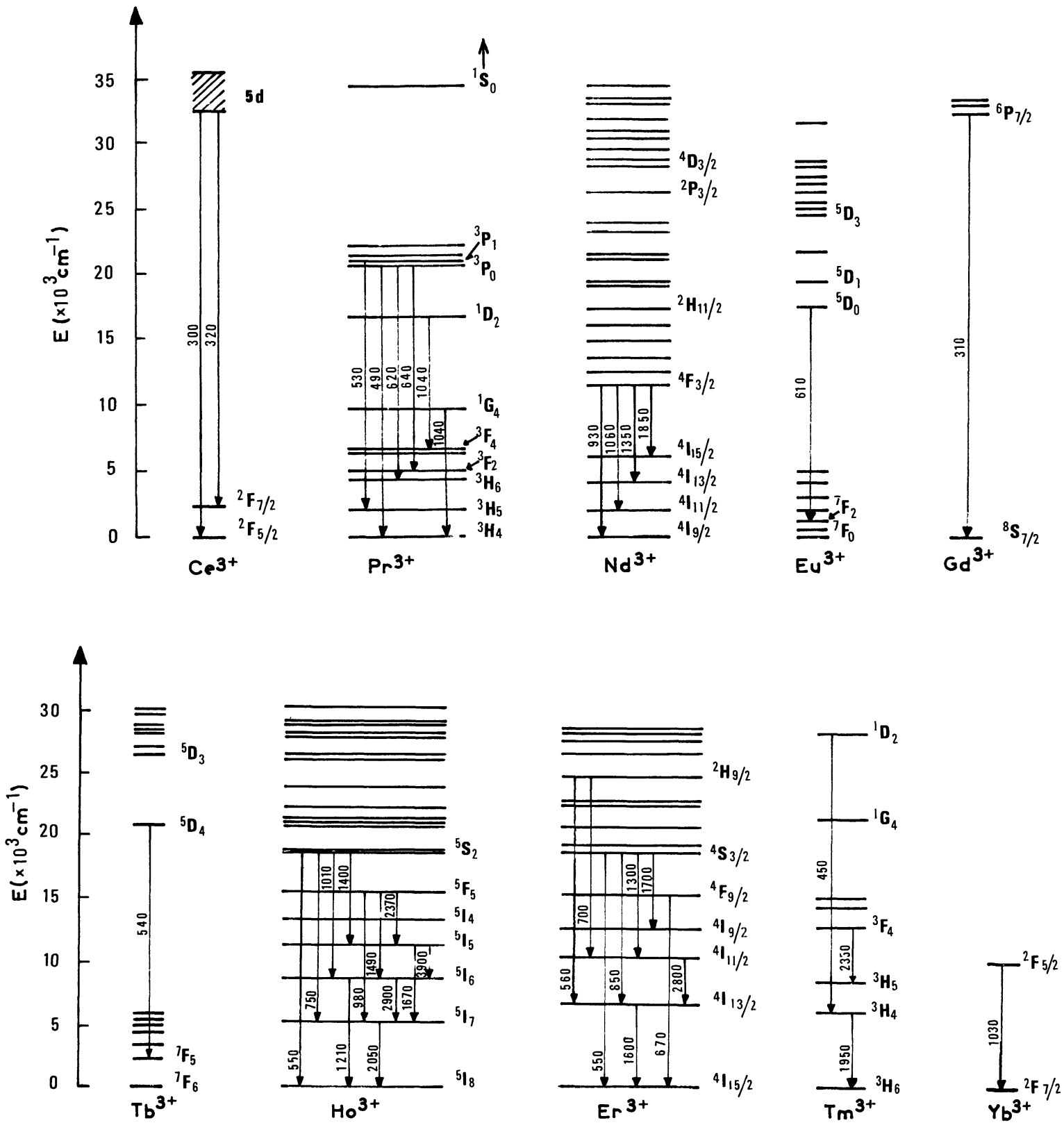

Figs. 13 et 14. - Diagramme des principaux niveaux d'énergie électroniques d'ions terre rare trivalents. Les longueurs d'onde des raies d'émission intervenant dans les luminophores et les matériaux lasers sont mentionnées en $\mathrm{nm}$.

[Diagram of the main electronic energy levels of trivalent rare earth ions. The wavelengths of the fluorescence lines occurring in luminophores and solid state laser materials are mentioned in $\mathrm{nm}$.]

$R_{0}$, distance critique, est proportionnelle au recouvrement spectral

$$
\int g_{\mathrm{S}}(\nu) \cdot g_{\mathrm{A}}(\nu) \mathrm{d} \nu
$$

$R_{0}$ est la distance pour laquelle la probabilité de transfert est égale à la probabilité d'émission de $S$.

$\mathrm{Ce}$ sont les profils des déclins de fluorescence de $\mathrm{S}$ en présence de plusieurs concentrations en activateurs A qui peuvent aider à connaître la nature exacte des interactions multipolaires par la théorie d'Inokuti et Hirayama [31] si on néglige la diffusion
S-S et par la théorie de Yokota et Tanimoto avec la diffusion [32].

La figure 17 représente des cas particuliers de transfert d'énergie que l'on rencontre fréquemment dans les terres rares. Il s'agit d'une part en a de la relaxation croisée, transfert partiel de l'énergie d'un état excité vers un ion voisin qui induit une extinction lorsque la résonance se produit entre 2 sous-niveaux excités et 2 sous-niveaux du fondamental. Les exemples les mieux connus sont les relaxations croisées ${ }^{5} \mathrm{D}_{3}-{ }^{5} \mathrm{D}_{4}$ et ${ }^{7} \mathrm{~F}_{6}-{ }^{7} \mathrm{~F}_{0}$ de $\mathrm{Tb}^{3+}$ et ${ }^{4} \mathrm{~F}_{3 / 2}{ }^{-4} \mathrm{I}_{15 / 2}$ et ${ }^{4} \mathrm{I}_{15 / 2}-{ }^{4} \mathrm{I}_{9 / 2}$ de $\mathrm{Nd}^{3+}$. D'autre part, nous avons aussi schématisé en $b$ et $c$ les mécanismes d'addition de photons par 


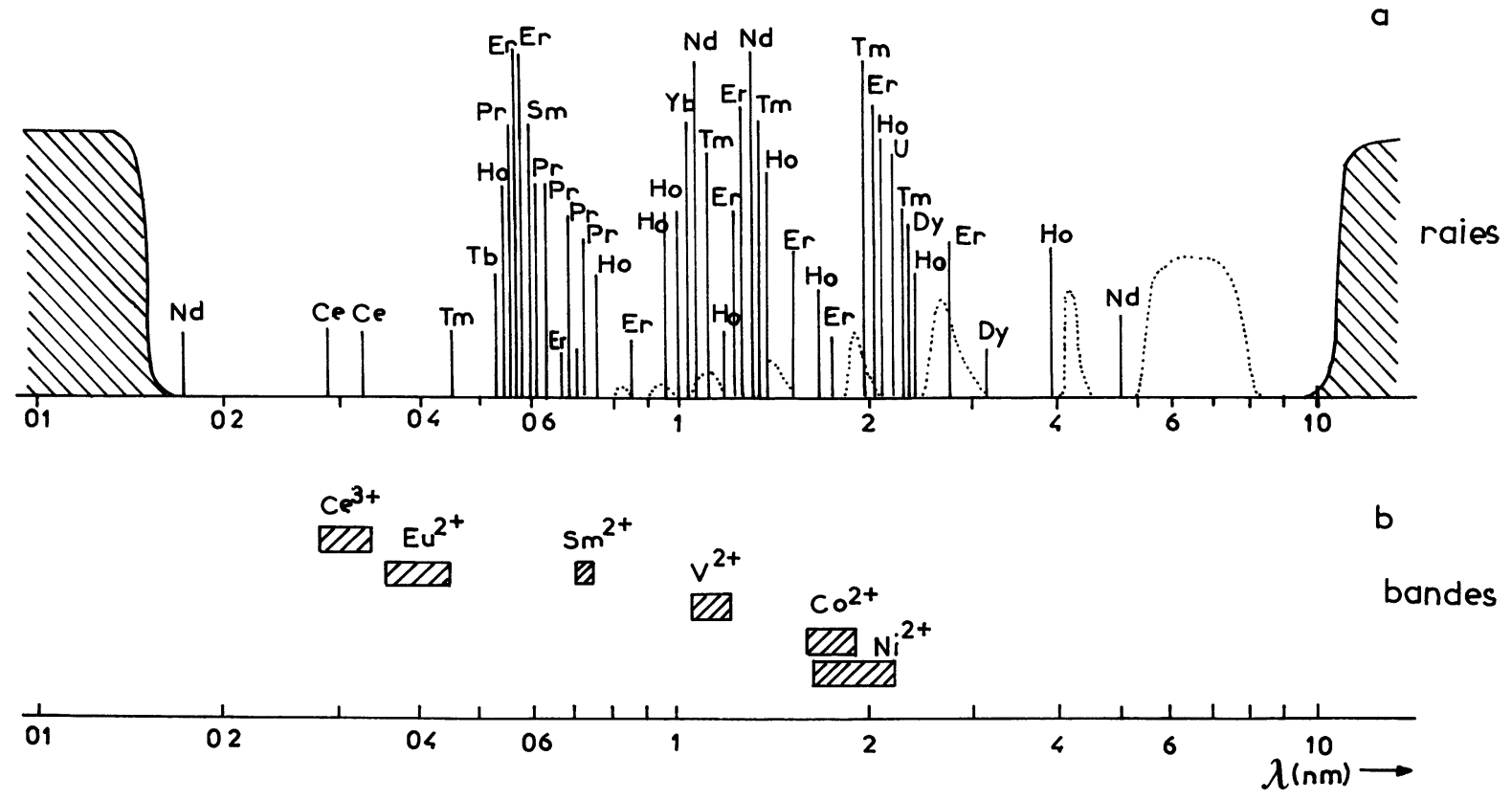

Fig. 15. - Position des principales raies et bandes émises par les ions activateurs dans la fenêtre de transmission des réseaux de base : a) raies des terres rares trivalentes, b) bandes des terres rares et des ions de transition. Les courbes en pointillés représentent les absorptions des éléments rencontrés dans l'atmosphère.

[Location of the main lines and of the mainbands emitted by activator ions in the vicinity of the transmission window of the host networks : a) sharp lines of trivalent rare-earth ions ; b) broad bands of rare-earth ions and metal transition ions. The discontinued spectra represent absorption of the principal elements of the atmosphere.]
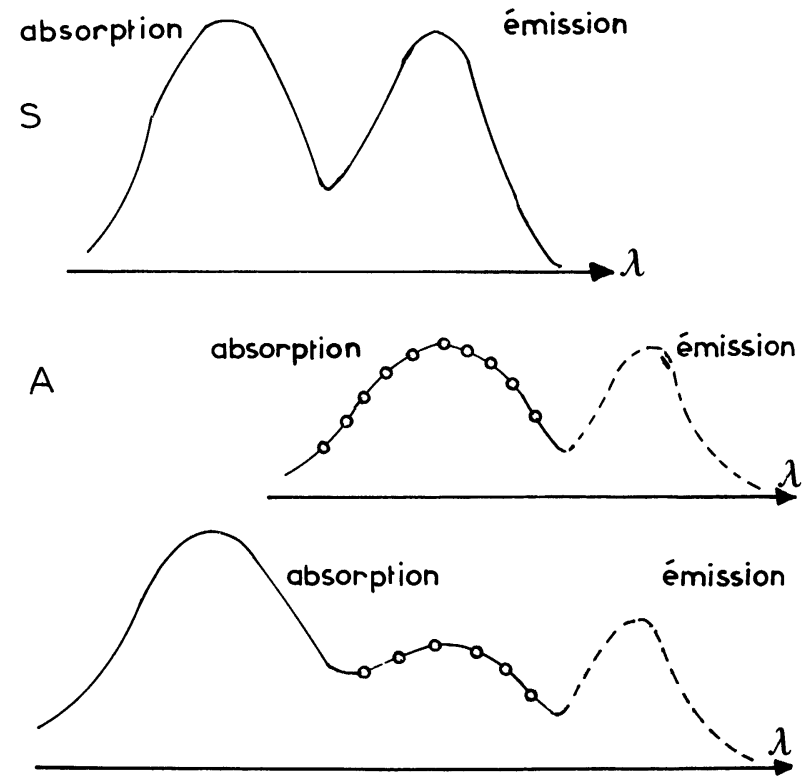
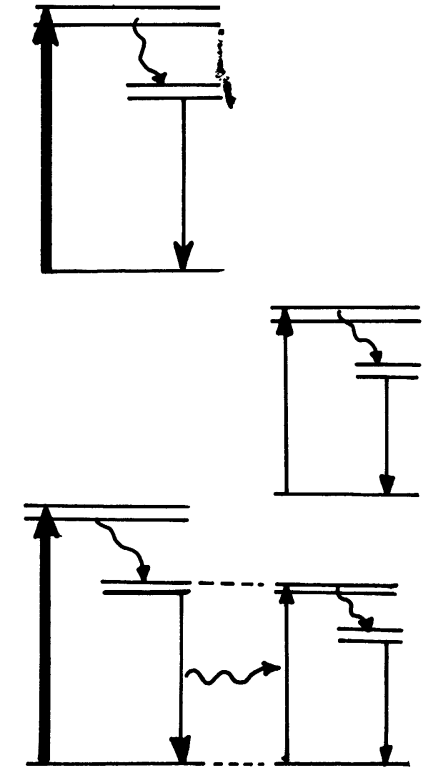

S

A

Fig. 16. - Transfert d'énergie entre un ion sensibilisateur $S$ et un ion activateur A présentant tous les deux un décalage de Stokes. Le spectre d'excitation de l'émission de A dans l'échantillon contenant $\mathrm{S}$ et $\mathrm{A}$ est alors confondu avec le spectre d'absorption respectivement de A seul et de $\mathrm{S}$ seul.

[Energy transfer between sensitizer ion $S$ and activator ion A characterized by a Stokes shift. The excitation spectrum of the A fluorescence studied in the sample codoped by $\mathrm{S}$ and $\mathrm{A}$ inverses with the absorption spectrum both of the $\mathrm{A}$ ion and the $S$ ion.] 


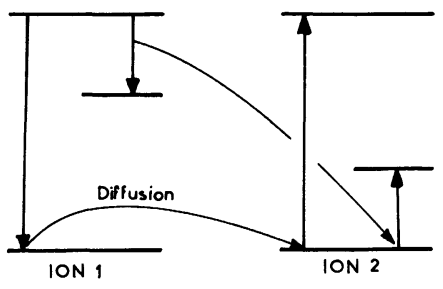

(a)
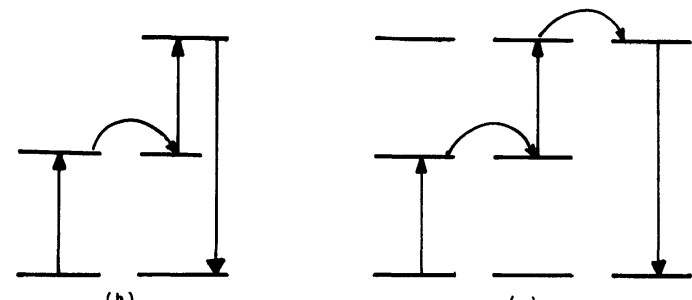

Fig. 17. - Modèles de transferts d'énergie couramment rencontrés avec les ions terre rare et les ions de transitions : a) diffusion et relaxation croisée entre les niveaux d'énergie de 2 ions pour lesquels la conservation des écarts énergétiques est respectée ; b) addition de photons par transfert d'énergie entre 2 ions ; c) addition de photons par sensibilisation coopérative entre 3 ions.

[Energy transfer models currently used with rare-earth ions and metal transition ions. a) diffusion and crossrelaxation between the energy levels of two ions for which the energetic gaps are respected ; b) energy transfer by addition of photons between two ions ; c) cooperative sensitization between three ions.]

transfert d'énergie entre 2 ou plusieurs ions (sensibilisation coopérative) générateurs de fluorescences anti-Stokes dans les ions terres rares pour lesquels on trouve aisément des résonances associées à des durées de vie de niveaux excités assez grandes. On rencontre ces mécanismes avec $\mathrm{Gd}^{3+}, \mathrm{Tb}^{3+}, \mathrm{Er}^{3+}$, $\mathrm{Tm}^{3+}, \mathrm{Yb}^{3+}[5,6]$. Dans les matériaux lasers soumis à des flux de pompage élevés, ils contribuent à abaisser le rendement de fluorescence.

L'extinction par concentration doit être liée aux différents types d'interaction entre les ions activateurs et bien sûr les impuretés qui peuvent piéger l'énergie excitatrice. Malgré les progrès récents dont a bénéficié ce domaine grâce aux techniques de spectroscopie-laser, la compréhension des mécanismes mis en jeux n'est pas maîtrisée et il convient d'approfondir les recherches.

\section{Interaction entre la recherche de luminophores et celle des lasers à solide.}

Jusqu'à ces dernières années il n'y a eu que peu de transferts de connaissances entre les deux principaux champs d'application des matériaux luminescents (les luminophores et les lasers à solide en raison, probablement, de la focalisation des recherches respectives sur les points essentiels mais différents comme le montre le tableau IV).

Plus récemment, on a assisté à un recouvrement des préoccupations de ces deux domaines. En effet, mentionnons en premier lieu l'apport considérable des instruments lasers, plus particulièrement des
Tableau IV. - Comparaison entre les pôles d'intérêts des luminophores et des matériaux lasers.

\section{Luminophores}

1. émission spontanée

2. chimie du solide polycristallin

3. pompage ultra-violet par 254 et $185 \mathrm{~nm} \mathrm{du} \mathrm{Hg}$ et d'énergie plus élevée

4. bandes de fluorencence raies fines proche infrarouge dans le visible lasers pompes YAG $-\mathrm{Nd}^{3}+$ doublé et triplé en fréquence associés à des lasers à colorants accordables dans l'uv, le visible et une partie de l'infrarouge, à l'analyse des matériaux luminescents polycristallins ou monocristallins. En second lieu, on peut dire qu'après les découvertes d'une part des lasers à solide $\mathrm{CaWO}_{4}\left(\mathrm{Nd}^{3+}\right)$, verre $\left(\mathrm{Nd}^{3+}\right)$ en 1961 et surtout de $\mathrm{Y}_{3} \mathrm{Al}_{5} \mathrm{O}_{12}\left(\mathrm{Nd}^{3+}\right)$ en 1964 et, d'autre part, la même année du luminophore $\mathrm{YVO}_{4}\left(\mathrm{Eu}^{3+}\right)$ pour la télévision en couleur et les lampes à vapeur de mercure corrigées, il y a eu une très forte poussée des études théoriques qui ont rejailli sur les deux domaines. Ce sont les déterminations des fameux diagrammes des niveaux d'énergie des ions terre rare trivalents et divalents et ceux des ions de transition. C'est aussi les travaux sur les relaxations radiatives et non radiatives internes aux terres rares et aujourd'hui des actinides. Enfin, signalons que le transfert des luminophores vers les lasers a été bénéfique pour tout ce qui touche aux phénomènes d'extinction par concentration et d'extinction thermique et que, depuis près de cinq ans, la recherche de nouveaux matériaux lasers accordables dans l'uv, le visible et le proche infrarouge, s'est orientée sur les ions de transition et de terre rare traditionnellement étudiés pour les produits du type luminophores à bandes larges. Citons le cérium trivalent $\mathrm{Ce}^{3+}$ dans $\mathrm{LaF}_{3}$ et $\mathrm{LiYF}_{4}$ (émission uv), le chrome trivalent $\mathrm{Cr}^{3+}$ dans l'alexandrite $\mathrm{BeAl}_{2} \mathrm{O}_{4}$, l'émeraude $\mathrm{Be}_{3} \mathrm{Al}_{2} \mathrm{Si}_{6} \mathrm{O}_{18}$ et les grenats, le titane trivalent $\mathrm{Ti}^{3+}$ dans le saphir, les ions de transition $\mathrm{Ni}^{2+}, \mathrm{Co}^{2+}, \mathrm{V}^{2+}$ dans les fluorures et les oxydes [1-3-7, 8]. De même, la tendance des recherches de nouveaux luminophores s'est orientée sur l'exploitation des raies étroites des terres rares de longueurs d'onde convenables, pour les composantes bleue $\left(\mathrm{Eu}^{2+}\right)$, verte $\left(\mathrm{Tb}^{3+}\right.$ après transferts $\left.\mathrm{Ce}^{3+} \rightarrow \mathrm{Gd}^{3+} \rightarrow \mathrm{Tb}^{3+}\right)$ et rouge $\left(\mathrm{Eu}^{3+}\right)$ des lampes trichromatiques de la nouvelle génération comme on le verra dans le paragraphe réservé aux luminophores.

7. Les monocristaux et les verres luminescents appliqués aux lasers, scintillateurs et concentrateurs solaires.

7.1 MATÉRIAUX LASERS. - Il existe essentiellement deux types de centres actifs pour l'effet laser : les 
centres à 3 niveaux représentés par $\mathrm{Al}_{2} \mathrm{O}_{3}\left(\mathrm{Cr}^{3+}\right)$ et les centres à 4 niveaux représentés par $\mathrm{Y}_{3} \mathrm{Al}_{5} \mathrm{O}_{12}\left(\mathrm{Nd}^{3+}\right)$ et tous les autres composés dopés par $\mathrm{Nd}^{3+}$. Le seuil de l'inversion de population est évidemment beaucoup plus facile à atteindre et dépasser avec 4 niveaux qu'avec 3 niveaux puisque la transition laser aboutit sur un niveau dépeuplé situé à $2000 \mathrm{~cm}^{-1}$ au-dessus du fondamental et non pas sur le fondamental comme cela est le cas avec le rubis. La figure 18 illustre bien cette différence de comportement en montrant les évolutions du coefficient d'amplification a $(\nu)$ en $\mathrm{cm}^{-1}$ en fonction de l'intensité de la source de pompage $\rho(\nu)$ dans les 2

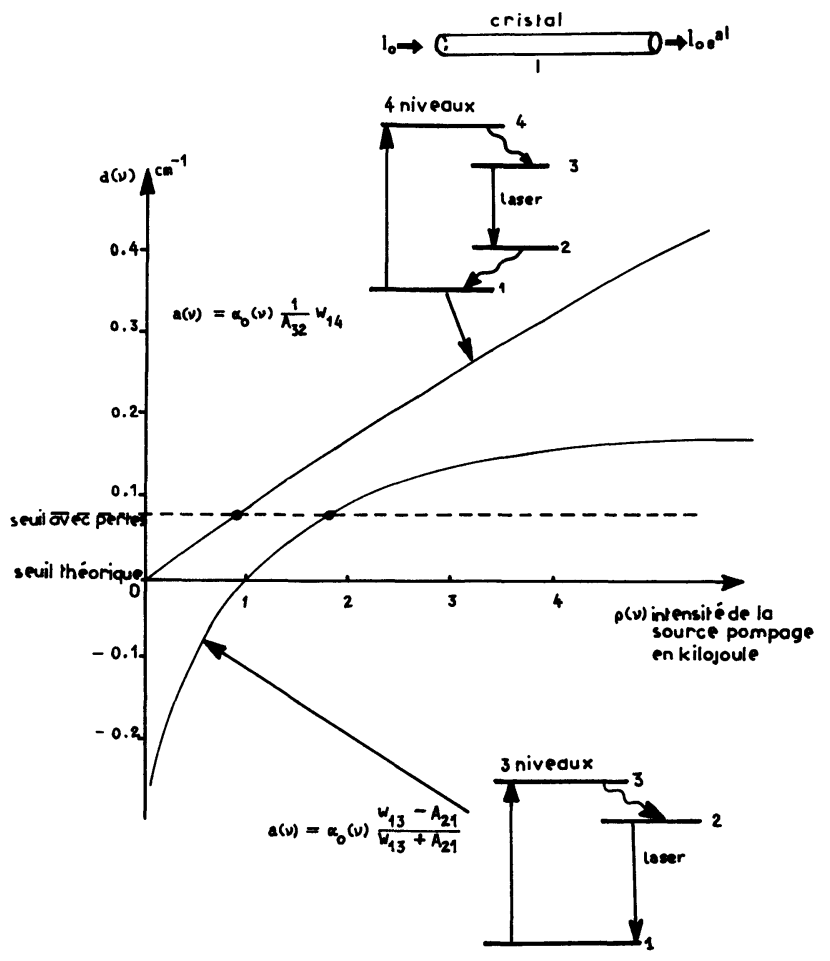

Fig. 18. - Variation du coefficient d'amplification $a(\nu)$ en fonction de l'intensité spectrale énergétique de la source $\rho(\nu)$ contenue dans l'expression $W_{i j}=B_{i j} \rho(\nu)$ où $B_{i j}$ désigne le coefficient d'absorption d'Einstein. Sur cette figure, $A_{i j}$ représente la probabilité d'émission spontanée égale à l'inverse de la durée de vie moyenne du niveau émetteur et $\alpha_{0}(\nu)$ correspond au coefficient d'absorption classique de la transition d'absorption lorsqu'on utilise des sources de faible intensité donc en admettant que la population du niveau fondamental est pratiquement égale à la concentration totale des ions actifs.

[Amplification coefficient $a(\nu)$ in versus of the energetic spectral intensity $\rho(\nu): W_{i j}=B_{i j} \rho(\nu)$ where $B_{i j}$ is the Einstein absorption coefficient. In the figure, $A_{i j}$ is the spontaneous emission probability equals the inverse of the average life time of the emitting level. $\alpha(\nu)$ is the standard absorption coefficient of the absorption transition by using weak intensity sources then by making the hypothesis that the population of the ground state is practically equal to the total concentration of activator ions.] cas. Sur cette figure on a utilisé les notations classiques de la spectroscopie et on a posé $W_{i j}=B_{i j} \cdot \rho(\nu)[4-8,9]$.

Trouver un bon cristal laser n'est pas chose facile si l'on note qu'après l'appariton du rubis en 1960 et du YAG $-\mathrm{Nd}^{3+}$ en 1964 on n'a pas trouvé de matériaux de substitution. En 20 ans, on n'a pu augmenter les performances du YAG $-\mathrm{Nd}^{3+}$ que d'un facteur 2. Doit-on considérer que c'est la preuve de la limitation de nos connaissances de la physico-chimie du solide ?

En réalité, les propriétés spectroscopiques exigées pour obtenir l'effet laser dans un solide comme par exemple : une forte absorption de la lumière excitatrice de préférence par des bandes larges, un rendement quantique de fluorescence élevé, une section efficace d'émission stimulée élevée et un profil spectral de raies fines pour les lasers de puissance, ne sont pas suffisantes. D'autres facteurs vont limiter considérablement le choix. Ce sont la qualité optique du cristal ou du verre, la résolution des problèmes difficiles de cristallogenèse d'échantillons de grandes dimensions (plusieurs $\mathrm{cm}^{3}$ ) et les propriétés de stabilité vis-à-vis de la lumière, de la chaleur, de l'eau et de la corrosion [9]. On trouvera dans la référence [8] les articles généraux des propriétés spectroscopiques des différentes classes de matériaux lasers.

7.2 SCINTIllateurs. - $\mathrm{NaI}\left(\mathrm{Tl}^{+}\right)$a tenu une place importante pendant près de trente ans pour les raisons suivantes : l'intensité de la sortie de lumière reste élevée, la résolution des impulsions est bonne, la réponse rapide, la densité et $Z$ sont grands et la sensibilité convenable. Cependant, le matériau est hygroscopique, délicat de mise en œuvre sous forme monocristalline et il subit des dommages notables après une exposition même modeste aux rayonnements ambiants.

On préfère, aujourd'hui, faire appel à la phase $\mathrm{Bi}_{4} \mathrm{Ge}_{3} \mathrm{O}_{12}$ des germanates de bismuth que l'on note BGO. Il est dense $\left(7,13 \mathrm{~g} \mathrm{~cm}^{-3}\right)$. La concentration en $\mathrm{Bi}^{3+}$, de $Z$ élevé, est importante. Il peut être utilisé avec des champs magnétiques intenses en physique nucléaire des hautes énergies. Sa longueur de radiation, inverse du coefficient d'absorption, égale à $1,12 \mathrm{~cm}$, est inférieure à près de la moitié de celle de $\mathrm{NaI}\left(\mathrm{Tl}^{+}\right)$. De même la phosphorescence est beaucoup plus faible que celle de $\mathrm{NaI}\left(\mathrm{Tl}^{+}\right)$.

De plus, il n'est pas hygroscopique, sa manipulation reste aisée, et, il est mieux . résistant aux dommages par radiations que $\mathrm{NaI}\left(\mathrm{Tl}^{+}\right)$.

Ses applications sont nombreuses et variées. Ainsi l'expérience $\mathrm{L}_{3}$ du LEP (Large Electron Positron) du CERN de Genève qui sera mise en place en 1988 en vue d'étudier l'interaction faible (boson Zo), nécessite la croissance de 12000 monocristaux de dimensions $24 \times 3 \times 3 \mathrm{~cm}^{3}$. La médecine nucléaire demande $\mathrm{BGO}$ pour la tomographie à émission de positrons (traceurs injectés dans le corps) à cause de sa bonne résolution spatiale. Mentionnons, enfin, 
les applications à la recherche du pétrole par détection des rayonnements émis par les couches géologiques.

Les propriétés optiques de BGO avaient été présentées en 1974 [10], mais la complexité des mécanismes d'absorption et d'émission n'avait pas été complètement élucidée. En raison de l'avenir prometteur de ce matériau et à la demande des expérimentateurs du CERN, nous en avons repris l'analyse spectroscopique par les techniques laser d'excitation sélective dans l'uv et de spectroscopie résolue dans le temps. Les résultats obtenus apportent un éclairage nouveau sur les interprétations de la photoluminescence de ce scintillateur [11].

Quant à $\mathrm{Y}_{3} \mathrm{Al}_{5} \mathrm{O}_{12}\left(\mathrm{Ce}^{3+}\right)$, il est développé sous forme monocristalline pour la fabrication d'écrans haute résolution et haute brillance de microscopes électroniques à balayages. En effet, la durée de vie du niveau de la configuration $5 \mathrm{~d}$ étant assez courte ( $\sim 80 \mathrm{~ns})$, on peut indexer le faisceau avec une résolution supérieure à $10^{7}$ images par seconde. Ce matériau sera probablement de plus en plus utilisé dans les tubes à analyseurs d'images.

7.3 CONCENTRATEURS SOLAIRES LUMINESCENTS. - Le principe de ces dispositifs est basé sur l'absorption des radiations solaires dans un collecteur vitreux contenant des ions fluorescents pour lesquels les bandes d'émission ont peu ou pas de recouvrement avec les bandes d'absorption pour éviter les transferts d'énergie dissipateurs du rayonnement de fluorescence. La fluorescence est piégée par réflexion totale sur les faces du collecteur puis concentrée sur les bords où l'on a déposé des cellules photovoltaïques. Les ions activateurs doivent être choisis pour que leurs raies de fluorescence coïncident avec le maximum de sensibilité spectrale de la photopile au silicium ( 800 à 900 nm) (Fig. 19).

Actuellement, deux voies de recherches se dégagent parmi les polymères organiques dopés par des espèces organiques fluorescentes et les verres (et même les cristaux) inorganiques plus stables dopés soit par $\mathrm{Nd}^{3+}$ et $\mathrm{Yb}^{3+}$ mettant à profit les transferts d'énergie $\mathrm{Nd}^{3+} \rightarrow \mathrm{Yb}^{3+}$ [12], soit par $\mathrm{Cr}^{3+}$ et $\mathrm{Nd}^{3+}$ [13] dans le but d'utiliser les transferts $\mathrm{Cr}^{3+} \rightarrow \mathrm{Nd}^{3+}$. Une recherche intensive est conduite d'abord avec le chrome dont la substitution sur des sites à champ cristallin faible induit les bandes larges ${ }^{4} \mathrm{~T}_{2} \rightarrow{ }^{4} \mathrm{~A}_{2}$ centrées entre $700 \mathrm{~nm}$ et $11000 \mathrm{~nm}$. Là encore les recherches devront être approfondies surtout pour les transferts $\mathrm{Cr}^{3+} \rightarrow \mathrm{Nd}^{3+}$ dont les résultats restent loin des espérances annoncées [13].

Dans les verres dopés par $\mathrm{Cr}^{3+}$, les transitions non radiatives de cet ion abaissent le rendement quantique de fluorescence. Les meilleurs résultats obtenus à ce jour sont 0,17 dans un verre silicate de lithium et de calcium [14] et 0,22 dans un verre phosphate de lanthane et de lithium $[15,16]$ tandis que dans les cristaux dopés par $\mathrm{Cr}^{3}+$ le rendement est égal à 1 à la température ambiante. Une solution intermédiaire a donc été proposée par la formation de vitrocéramiques en soumettant des verres conte-
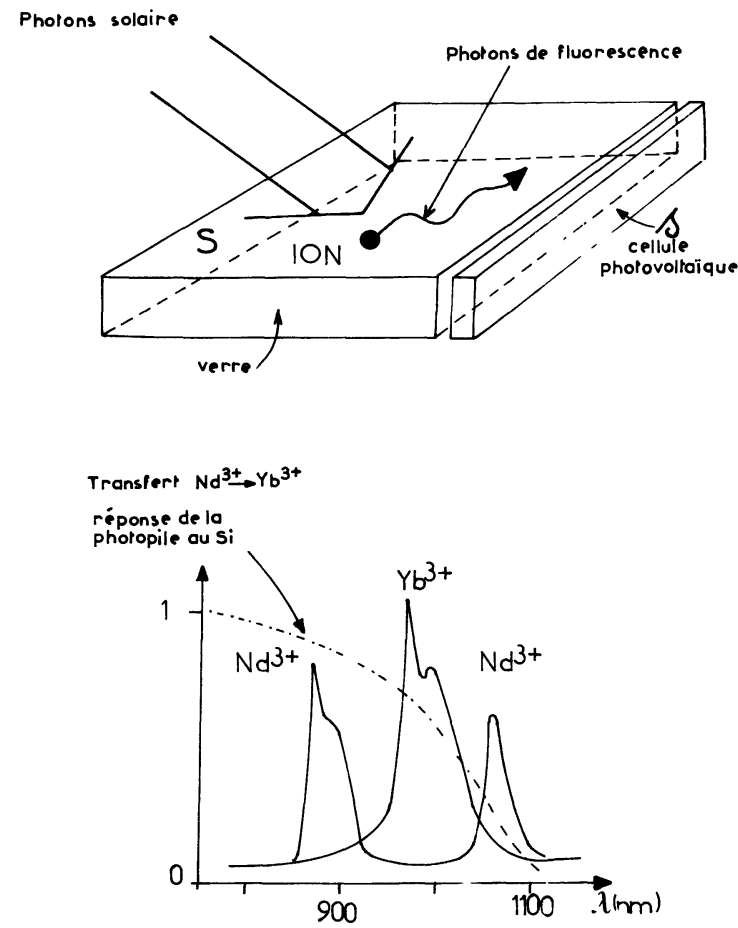

Fig. 19. - Schéma de principe du fonctionnement d'un concentrateur solaire luminescent. L'ion activateur est l'ion dopant du support vitreux soumis au rayonnement solaire. Les photons de fluorescence sont collectés par la cellule photovoltaïque au silicium dont la réponse doit coïncider avec le spectre d'émission de l'activateur. Les spectres représentés correspondent au cas du transfert d'énergie $\mathrm{Nd}^{3+} \rightarrow \mathrm{Yb}^{3+}$.

[Luminescent solar concentrator device. The fluorescent ion dopes the glassy support and it absorbs the solar beam. Fluorescence photons are collected by photovoltaic cell for which the spectral response must coincide with the activator emission spectrum. The emission spectra shown in figure are associated with the $\mathrm{Nd}^{3+} \rightarrow \mathrm{Yb}^{3+}$ energy transfer.]

nant du chrome à des traitements thermiques appropriés proches de la température de cristallisation [1720].

L'ion $\mathrm{Cr}^{3+}$ joue alors les rôles d'agent nucléant et d'ion luminescent. Par exemple les dimensions des microcristallites formés dans la cordiérite varient de 40 à $400 \AA$ donc nettement inférieures aux longueurs d'onde du visible ce qui évite des diffusions gênantes. Les rendements du chrome dans les vitrocéramiques, formées à partir de la cordiérite, de la gahnite, du $\beta$ quartz et de la pétalite, sont nettement améliorés $(\sim 1$ avec la gahnite, 0,5 dans le $\beta$-quartz et 0,75 dans la pétalite). Ajoutons que la préparation de ces nouveaux matériaux nous servent à étudier les phénomènes de nucléation et que les recherches en cours révèlent des microcristallites de structure spinelle $\mathrm{MgAl}_{2-x} \mathrm{Cr}_{x} \mathrm{O}_{4}$ ou $\mathrm{ZnAl}_{2-x} \mathrm{Cr}_{x} \mathrm{O}_{4}$ de teneurs en chrome variables [21].

Les perspectives des concentrateurs solaires dépendent de leur rendement lumineux, du décalage de Stokes entre l'absorption et l'émission et aussi 
beaucoup de leur stabilité. Toutes ces conditions peuvent être réalisées avec les vitrocéramiques dopées par $\mathrm{Cr}^{3+}$ et rajoutons que ce sont les mêmes critères qui sont nécessaires à la recherche de matériaux lasers accordables. Les phases de gahnite et de pétalite sont les plus prometteurs lorsque la nucléation a pour origine $\mathrm{Cr}^{3+}$ ou $\mathrm{Zr}^{4+}$.

\subsection{LES COMPOSÉS LUMINESCENTS POLYCRISTAL} LINS.

7.4.1 Luminophores pour tubes fluorescents. - On pourrait penser qu'une bonne approche de la recherche de luminophores consiste à sélectionner un ion activateur dont on connaît le spectre d'émission, puis de l'incorporer dans un réseau cristallin hôte de symétrie convenable et dont le rendement de fluorescence est élevé. Comme les raies excitatrices du mercure sont 185 et $254 \mathrm{~nm}$, on pourrait aussi modifier les paramètres de la maille et introduire une forte absorption dans cette région ultraviolette de telle sorte que le transport d'énergie soit efficace entre la matrice et l'activateur. Une autre solution intéressante peut être fournie par la mise en œuvre de transfert d'énergie entre un ion sensibilisateur et l'ion activateur évoqué précédemment. Il existe, en fait, des centaines de composés qui remplissent tous ces critères et la pratique montre que le bon luminophore doit satisfaire à d'autres exigences qui limitent considérablement les candidats. Par exemple, ils doivent conserver une excellente stabilité

Tableau V

Oxydes de : Lanthane cérium

Yttrium

Prix :

1

6

On remarque qu'il serait intéressant de trouver des ions activateurs de substitution à l'europium trivalent pour la composante rouge et au terbium trivalent pour la composante verte. Pour cette dernière, il y a coïncidence des objectifs d'augmentation du rendement de fluorescence par transfert d'énergie et de diminution du coût par l'adjonction dans les aluminates de cérium $\mathrm{Ce}^{3+}$ avec le terbium $\mathrm{Tb}^{3+}$.

7.4.2 Evolution des luminophores pour l'éclairage. - Tubes des deux premières générations. Les premiers tubes sortis en 1938 utilisaient les minerais pulvérisés de $\mathrm{CaWO}_{4}$ et $\mathrm{MgWO}_{4}$. La synthèse des luminophores dans les laboratoires date de 1940 et les premiers dopages furent $\mathrm{Pb}^{2+}$ dans $\mathrm{MgWO}_{4}$, $\mathrm{Mn}^{2+}$ dans $\mathrm{Zn}_{2} \mathrm{SiO}_{4}$ ou 2 CdO- $\mathrm{B}_{2} \mathrm{O}_{3}$. Le démarrage de la production industrielle des tubes s'est produit pendant la guerre mais il a fallu attendre 1948 pour voir apparaître les tubes développés en Angleterre avec les fameux halophosphates de calcium et de strontium dopés par $\mathrm{Sb}^{3+}$ et $\mathrm{Mn}^{2+}$. face aux attaques des atomes de mercure et des radiations de courte longueur d'onde émises par le mercure. Ces conditions sont d'autant plus difficiles à réaliser que les dimensions des nouveaux tubes et nouvelles lampes sont de plus en plus réduites. De plus, le rendu des couleurs et l'indice du triangle des couleurs doivent être satisfaisants. Il faut également éviter les absorptions parasites dans le visible où émettent les composés. Ces bandes d'absorption sont créées par la formation de centres colorés sous l'excitation par les raies du mercure dans l'uv lointain, lesquelles peuvent ausi casser les liaisons chimiques et changer la valence de l'activateur. Ces changements de valence peuvent intervenir avec le manganèse $\left(\mathrm{Mn}^{2+} \rightarrow \mathrm{Mn}^{3+}\right)$, le cuivre $\left(\mathrm{Cu}^{+} \rightarrow \mathrm{Cu}^{2+}\right)$, le titane $\left(\mathrm{Ti}^{3+} \rightarrow \mathrm{Ti}^{4+}\right)$ et le cérium $\left(\mathrm{Ce}^{3+} \rightarrow \mathrm{Ce}^{4+}\right)$. La granulométrie de la poudre déposée sur l'enveloppe du tube doit assurer un maximum d'émission à des températures de plus en plus élevées $\left(50^{\circ} \mathrm{C}\right.$ dans les tubes anciens et $150^{\circ} \mathrm{C}$ pour les nouveaux). Les autres problèmes posés par l'interface entre la poudre luminescente et le milieu gazeux sont la formation de complexes mercuriques avec le sodium, la diffusion des ions sodium du verre sur le luminophore et l'équilibre qui s'établit entre les électrons diffusants sur la surface et les ions mercure [22, 23]. Enfin, n'oublions pas les facteurs économiques qui, en fin de compte, imposent le choix définitif. A titre d'information, le tableau $\mathrm{V}$ indique les prix relatifs des oxydes de terres rares [24]

$\begin{array}{ccc}\text { Gadolinium } & \text { Terbium } & \text { Europium } \\ 10 & 85 & 110\end{array}$

Les tubes de la deuxième génération étaient nés et devaient être exploités jusque vers les années 80 . Leurs performances ont peu évolué puisque, par exemple, le tube F40 T12 construit aux USA ( $40 \mathrm{~W})$ appelé " blanc industrie ", caractérisé par une température de couleur de $4500 \mathrm{~K}$, produisait respectivement 2360 lumens en 1949 et 3200 lumens en 1968.

Cet halophosphate de calcium codopé par $\mathrm{Sb}^{3+}$ et $\mathrm{Mn}^{2+}$, de structure apatite admet les formules :

$$
\mathrm{Ca}_{5}\left(\mathrm{PO}_{4}\right)_{3} \mathrm{~F} ; \quad \mathrm{Ca}_{5}\left(\mathrm{PO}_{4}\right)_{3} \mathrm{Cl} ; \mathrm{Ca}_{5}\left(\mathrm{PO}_{4}\right)_{3} \mathrm{~F}, \mathrm{Cl}
$$

La limitation de l'optimisation est due à la formation de centres colorés appelés $\mathrm{E}_{\mathrm{II}}$ dont on peut réduire la concentration par addition de cadmium qui absorbe $185 \mathrm{~nm}$ mais pas $254 \mathrm{~nm}$. Mais l'interruption de l'usage du cadmium, élément toxique et cher, nécessite une telle réduction des défauts dans les produits de départ qu'il a fallu envisager de trouver d'autres luminophores. 
- Les tubes trichromatiques de la $3^{\mathrm{e}}$ génération.

La solution est venue avec le mélange de 3 luminophores bleu, vert et rouge commercialisé vers 197980. Les activateurs ont été sélectionnés de telle sorte que leurs spectres recouvrent assez bien la courbe de sensibilité spectrale de l'œil. La figure 20 montre les spectres d'émission des 3 luminophores les plus utilisés [25] :

$$
\begin{array}{ll}
\mathrm{BaMg}_{2} \mathrm{Al}_{16} \mathrm{O}_{27}\left(\mathrm{Eu}^{2+}\right) & \text { composante bleue } \\
\mathrm{Ce}_{0,68} \mathrm{MgAl}_{11} \mathrm{O}_{19}\left(0,32 \mathrm{~Tb}^{3+}\right) & \text { composante verte } \\
\mathrm{Y}_{2} \mathrm{O}_{3}\left(\mathrm{Eu}^{3+}\right) & \text { composante rouge }
\end{array}
$$

Le succès de ces oxydes est dû à leur bonne stabilité dans l'environnement du tube à vapeur de mercure : il n'y a ni halogène comme dans l'halophosphate, ni groupement phosphate et, de plus, ce sont des produits réfractaires dont la température de préparation est de l'ordre de $1500^{\circ} \mathrm{C}$. Malgré son coût élevé, le terbium s'est imposé pour la composante verte donnée par l'aluminate. Son rival, $\mathrm{Zn}_{2} \mathrm{SiO}_{4}\left(\mathrm{Mn}^{2+}\right)$ est peu cher, admet une bonne réponse spectrale mais est beaucoup moins stable. Les transferts d'énergie $\mathrm{Ce}^{3+} \rightarrow \mathrm{Tb}^{3+}$ sont efficaces et on essaie de les améliorer par l'addition de gadolinium $\mathrm{Gd}^{3+}$ ou de bismuth $\mathrm{Bi}^{3+}$ et donc de diminuer la teneur en $\mathrm{Tb}^{3+}$. La bande d'absorption ${ }^{2} \mathrm{~F}_{5 / 2} \rightarrow{ }^{5} \mathrm{~d}$ de $\mathrm{Ce}^{3+}$ a une force d'oscillateur élevée puisqu'elle correspond à une transition permise interconfigurationnelle et une partie coïncide avec la raie $185 \mathrm{~nm}$ du mercure. La diffusion $\mathrm{Ce}^{3+} \rightarrow \mathrm{Ce}^{3+}$ permet d'atteindre les ions $\mathrm{Tb}^{3}+$ qui acceptent l'énergie à la fois par la transition permise ${ }^{7} \mathrm{~F}_{6} \rightarrow{ }^{5} \mathrm{~d}$ et les transitions internes à la configuration $4 \mathrm{f}$. Les mécanismes de transferts d'énergie seraient de nature multipolaire du type dipôle-quadrupôle ce qui explique le besoin en $\mathrm{Tb}^{3+}$ de teneur suffisamment grande pour que les distances $\mathrm{Ce}^{3+} \rightarrow \mathrm{Tb}^{3+}$ soient courtes.

- Perspectives sur de nouveaux luminophores ( $4^{\mathrm{e}}$ génération).

Une nouvelle famille de luminophores vient d'apparaître au point d'être en compétition directe avec les aluminates dopés par $\mathrm{Eu}^{3+}$ et $\mathrm{Tb}^{3+}$, ce sont des borates de formule $\mathrm{LnMgB}_{5} \mathrm{O}_{10}(\mathrm{Ln}=$ terre rare) $[26,27]$. Ils représentent une bonne stabilité malgré une température de synthèse basse $\left(\sim 1000^{\circ} \mathrm{C}\right)$. Le principal avantage de cette température est économique : les pertes d'activateurs par volatilisation sont négligeables. Les sites $\mathrm{Ln}^{3+}$ et $\mathrm{Mg}^{2+}$ acceptent des cations de substitution trivalents et bivalents et on retrouve les mêmes possibilités de transfert d'énergie qu'avec les aluminates. De plus, les connaissances acquises depuis longtemps sur la chimie des borates permettent de jouer sur les modifications graduelles des paramètres du réseau donc sur l'évolution des spectres de fluorescence. On ne sait pas encore quel sera l'impact technologique de ces nouveaux composés mais déjà en 1985 on annonçait les tubes de la quatrième génération (diamètre $\varnothing=26 \mathrm{~mm}$, plasma d'argon et de krypton) [28] dans lesquels on a supprimé $\mathrm{Eu}^{3+}$ en adoptant le manganèse $\mathrm{Mn}^{2+}$ pour la composante rouge dans $\left(\mathrm{Ce}^{3+}, \mathrm{Gd}^{3+}\right)\left(\mathrm{Mg}^{2+}, \mathrm{Mn}^{2+}\right) \mathrm{B}_{5} \mathrm{O}_{10}$, en conservant le terbium $\mathrm{Tb}^{3+}$ pour la composante verte dans

$$
\left(\mathrm{Ce}^{3+}, \mathrm{Gd}^{3+}, \mathrm{Tb}^{3+}\right)\left(\mathrm{Mg}^{2+}, \mathrm{Mn}^{2+}\right) \mathrm{B}_{5} \mathrm{O}_{10}
$$

tout en bénéficiant de transferts supplémentaires du site trivalent vers le site bivalent et en ajoutant $\left(\mathrm{Sr}^{2+}, \mathrm{Eu}^{2+}\right) \mathrm{Al}_{14} \mathrm{O}_{25}$ pour la composante bleue. On est même revenu à un mélange supplémentaire en ajoutant des halophosphates de calcium dopés soit par $\mathrm{Sb}^{3+}$, soit par $\mathrm{Mn}^{2+}$. L'avenir dira si ces borates remplaceront ou pas les aluminates.

L'intérêt cristallographique des borates est que les terres rares $\mathrm{Ln}^{3+}$ appartiennent à des chaînes de polyèdres asymétriques à arêtes communes qui forment des zig-zag (distance La-La intra-chaîne = $4 \AA$; distance La-La inter-chaîne $=6,4 \AA$ ) et qui confèrent au matériau un caractère quasi dimensionnel spécialement dans les composés concentrés à base d'europium, ou de terbium ou de gadolinium (30).

\section{- Les lampes compactes.}

On les a vu apparaître sur le marché en 1981 avec des diamètres de tubes très faibles $(\varnothing=10 \mathrm{~mm})$ ce qui augmente la température interne jusqu'à $150^{\circ} \mathrm{C}$. Les luminophores doivent donc résister à cette température. Un exemple de composés utilisés dans ces lampes est donné ci-dessous :

$$
\begin{array}{ll}
3 \mathrm{Sr}_{3}\left(\mathrm{PO}_{4}\right)_{2}-\mathrm{CaCl}_{2}\left(\mathrm{Eu}^{2+}\right) & \text { bleu } \\
\mathrm{LaPO}_{4}\left(\mathrm{Ce}^{3+}, \mathrm{Tb}^{3+}\right) & \text { vert } \\
\mathrm{Y}_{2} \mathrm{O}_{3}\left(\mathrm{Eu}^{3+}\right) & \text { rouge }
\end{array}
$$

L'objectif principal est de remplacer les lampes à incandescence mais ces lampes compactes restent chères et peu esthétiques. Cependant, cet axe de recherche est justifié par la durée de vie et l'efficacité lumineuse accrue des lampes compactes par rapport aux lampes à incandescence : une lampe à incandescence de $60 \mathrm{~W}$ admet une durée de vie de $1000 \mathrm{~h}$ avec une efficacité de 15 lumens par watt tandis qu'un tube fluorescent de $40 \mathrm{~W}$ fonctionne $20000 \mathrm{~h}$ avec une efficacité de 80 lumens par watt.

En conclusion, la méthodologie de la recherche de luminophore pour l'éclairage n'est pas parfaitement définie. Parmi les premières synthèses on a rapidement découvert l'halophosphate de calcium codopé et toutes les tentatives amorcées pour trouver d'autres composés de même structure ou de structure voisine, qui sont de plus réfractaires, ont échoué [29]. Il est même surprenant de constater, aujourd'hui, que les nouveaux borates ne sont pas classés parmi les réfractaires. Probablement que les terres rares continueront de jouer un rôle capital parmi les activateurs mais les anciens dopants 


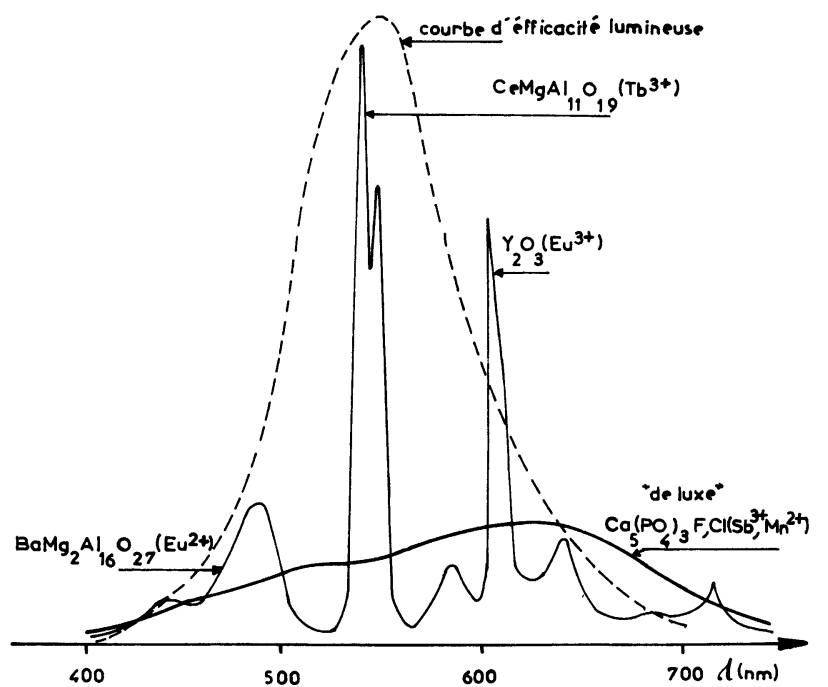

Fig. 20. - Correspondance entre les spectres d'émission des luminophores des lampes trichromatiques (composantes bleue, verte et rouge) et de la lampe " de luxe " à l'halophosphate avec la courbe d'efficacité lumineuse de l'œil.

[Emission spectra both of the three luminophores used in the trichromatic tubes (blue, green and red componants) and the "De Luxe " lamp. We also show the luminous efficiency curve of the eye.]

comme $\mathrm{Sb}^{3+}, \mathrm{Mn}^{2+}$, et aussi $\mathrm{Bi}^{3+}$ ne sont pas abandonnés. Enfin, mentionnons de nouveau, l'impact des études de transfert d'énergie sur le développement industriel de ces matrices et plus particulièrement dans celles pouvant accueillir plusieurs ions de valences variées et représentant un caractère unidimensionnel dans des chaînes ayant peu d'interaction commune [30].

7.4.3 Luminophores pour la télévision couleur. Les trois composés bleu, vert et rouge déposés sur les écrans de tubes cathodiques sont l'un des éléments de l'ensemble de la chaîne que constitue un récepteur TV et qui atteint de nos jours une perfection technique remarquable.

La bande bleue de $\mathrm{ZnS}\left(\mathrm{Al}^{3+}, \mathrm{Ag}^{+}\right)$convient bien. Ses deux concurrents contenant des terres rares sont $\mathrm{ZnS}\left(\mathrm{Tm}^{3+}\right)$ et $\mathrm{Sr}_{5}\left(\mathrm{PO}_{4}\right)_{3} \mathrm{Cl}\left(\mathrm{Eu}^{2+}\right)$ mais le premier a une raie mal adaptée vers le bleuvert tandis que le rendement de l'autre est trop faible.

L'œil est très sensible dans le vert et il n'est pas nécessaire de vouloir imposer un ion à spectre fin comme $\mathrm{Tb}^{3+}$. Le sulfure $(\mathrm{Zn}, \mathrm{Cd}) \mathrm{S}\left(\mathrm{Cu}^{+}, \mathrm{Al}^{3+}\right)$ bien connu admet une bande verte qui cadre parfaitement avec le domaine spectral souhaité. Bien que la linéarité de la réponse des composés à base d'ions terre rare aux densités de courant élevées est supérieure à celle des sulfures $(\mathrm{Zn}, \mathrm{Cd}) \mathrm{S}$, c'est le manque de stabilité vis-à-vis de l'eau qui entrave l'utilisation de $\mathrm{CaS}\left(\mathrm{Ce}^{3+}\right)$.

Quant à la composante rouge, elle est donnée par $\mathrm{Y}_{2} \mathrm{O}_{2} \mathrm{~S}\left(\mathrm{Eu}^{3+}\right)$ et sa raie intense vers $612 \mathrm{~nm}$ $\left({ }^{5} \mathrm{D}_{0} \rightarrow{ }^{7} \mathrm{~F}_{2}\right)$ car la bande de $(\mathrm{Zn}, \mathrm{Cd}) \mathrm{S}\left(\mathrm{Ag}^{+}\right)$est mal détectée par l'œil dans le rouge. Les autres candidats sont $\mathrm{YVO}_{4}\left(\mathrm{Eu}^{3+}\right)$, premier luminophore dopé par une terre rare en $1964, \mathrm{Y}_{2} \mathrm{O}_{3}\left(\mathrm{Eu}^{3+}\right)$ comme dans les lampes et $\mathrm{Mg}_{0.90} \mathrm{Ca}_{0.10} \mathrm{~S}\left(\mathrm{Eu}^{3+}\right)$ mais celui-ci n'est pas stable vis-à-vis de l'eau.

Les tendances de recherche actuelles sont dirigées vers des produits réfractaires qui supportent les accroissements de température causés par l'impact des rayons cathodiques sous des densités de courant élevées tout en conservant des réponses linéaires [33].

7.4.4 Les luminophores pour écrans renforçateurs de rayons $X$. - Ils sont élaborés en fonction de deux objectifs : diminuer le temps d'exposition du patient face aux rayons $X$ et éviter l'utilisation de sels d'argent coûteux dans les émulsions photographiques.

Vers les années 70 , on ne connaissait que $\mathrm{CaWO}_{4}$. Maintenant, ce sont encore les terres rares qui sont utilisées dans des réseaux denses où l'absorption des rayons $\mathrm{X}$ est grande. Les émissions les mieux adaptées au domaine de sensibilité optimale de l'émulsion sont celles de $\mathrm{Tb}^{3+}$ dans $\mathrm{LaOBr}, \mathrm{Gd}_{2} \mathrm{O}_{2} \mathrm{~S}, \mathrm{YTaO}_{4}$ et de $\mathrm{Eu}^{2+}$ dans $\mathrm{BaFCl}$. La radiographie à contraste de couleur sera probablement liée au développement de composés à base de $\mathrm{Gd}^{3+}, \mathrm{La}^{3+}$ et de $\mathrm{Ba}^{2+}$ absorbant de façon différente les rayonnements.

\subsubsection{Luminophores pour dispositifs électrolumines-} cents en couches minces. - La figure 21 représente une coupe schématique d'un écran plat avec les électrodes d'aluminium [1] sur lesquelles on applique $\sim 100 \mathrm{~V}$, les couches isolantes $\mathrm{Y}_{2} \mathrm{O}_{3}(3000 \AA)$ [2 et 4], la couche active d'épaisseur $5000 \AA$, la couche transparente conductrice [6] et le support en verre [5]. La meilleure matrice hôte est $\mathrm{ZnS}$ en raison de sa bande interdite suffisamment grande pour que les ions activateurs émettent des radiations visibles et aussi parce que sa constante diélectrique faible

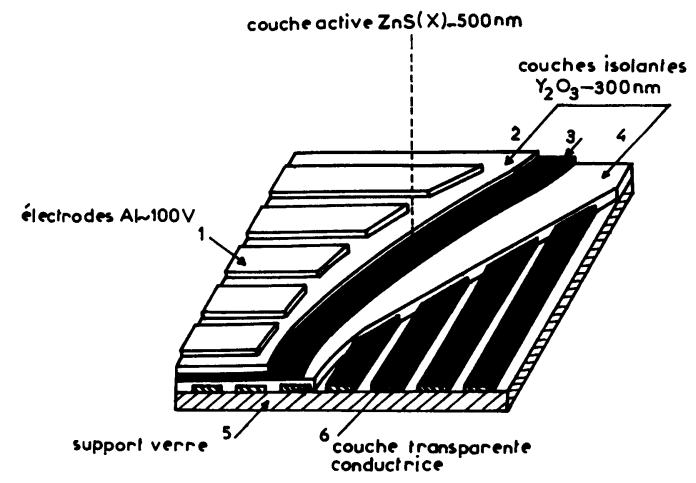

Fig. 21. - Coupe schématique d'un dispositif électroluminescent en couches minces.

[Schematic representation of the electroluminescent thin film device.] 
assure une bonne répartition de la tension entre la couche et les isolants. Ainsi $\mathrm{ZnS}\left(\mathrm{Mn}^{2+}\right)$ émet une bande large jaune vers $585 \mathrm{~nm}$ attribuée à la transition ${ }^{4} \mathrm{~T}_{2}\left({ }^{4} \mathrm{G}\right) \rightarrow{ }^{6} \mathrm{~A}_{1}\left({ }^{6} \mathrm{~S}\right), \mathrm{ZnS}\left(\mathrm{TbF}_{3}\right)$ dans le vert, $\mathrm{ZnS}\left(\mathrm{SmF}_{3}\right)$ dans le rouge et $\mathrm{SrS}\left(\mathrm{Ce}^{3+}\right)$ dans le bleu. Actuellement la composante verte est en cours de développement et les autres citées sont au stade des recherches.

\section{Conclusion.}

Les matériaux luminescents sont des éléments essentiels de la technologie moderne tant au niveau des appareils de grande consommation (éclairage, télévision, écrans d'ordinateurs) que de l'instrumentation spécifique de très haute performance (lasers, scintillateurs).

Pour chacune des applications présentées dans cet article nous avons indiqué l'état de l'art et les perspectives de développement. On essaie de tirer le profit maximum des transferts d'énergie entre deux ou plusieurs activateurs parmi les ions terre rare, les ions de transition et les ions lourds mais les modèles d'interaction et les mécanismes dynamiques entre les états excités ne sont pas encore maîtrisés. Il semble que l'action du gadolinium et du bismuth comme sensibilisateurs intermédiaires soit prépondérante pour le développement des luminophores. Indépendamment de l'étude des propriétés optiques des matériaux l'analyse de leur comportement en fonctionnement réel sous les effets des températures élevées, des densités de courants importantes, des décharges électriques du mercure, de l'impact des rayons cathodiques et de la linéarité de leur réponse doit être examinée systématiquement. L'expérience des industriels des luminophores montre que les problèmes soulevés ne sont pas résolus simplement et souvent l'empirisme domine.

Les progrès apportés par les techniques de spectroscopie laser de très haute résolution comme la spectroscopie par sélection de sites ou résolue dans le temps, la mesure des absorptions dans les états excités, l'absorption à 2 photons, l'addition de photons par transferts d'énergie sont incontestables mais il convient de poursuivre ces axes de recherche dans le but de prédire des modèles concernant l'influence des structures cristallines sur les transitions radiatives et non radiatives des ions actifs.

\section{Bibliographit}

[1] Kaminskil, A. A., Laser crystals, Their Physics and Properties (Springer-Verlag, Berlin) 1981.

[2] Weber, M. J., Handbook of Laser Science and Technology (C.R.C. Press Inc., Boca Raton, Florida) 1982.

[3] Tunable Solid State Lasers, Ed. P. Hammerling, A. B. Budgor and A. Pinto (Springer-Verlag, Berlin) 1985.

[4] Boulon, G., Principe de base des lasers, Chapitre I de l'ouvrage « Lasers et Industries de transformation » (Ed. Lavoisier TEC et DOC) 1986.

[5] Energy Transfer Processes in Condensed Matter, Ed. B. Di Bartolo (Plenum Press, New York) B 114 (1984). Cours présentés à l'Ecole d'Eté OTAN d'Erice (Sicile) Juin 1983. Parmi les cours intéressant les matériaux inorganiques, mentionnons :

- Introduction to energy transfer and relevant solid-state concepts (J. Bernard, D. Berry and F. Williams).

- Energy transfert among ions in solids (B. Di Bartolo).

- Mathematical methods for the description of energy transfer (V. Kenkre).

- Energy transfer in insulating materials (G. Blasse).

- Energy transfer and localization in ruby (G. Imbusch).

- Energy transfer and ionic solid state lasers (F. Auzel).

- Energy transfer between inorganic ion in glasses (R. Reisfeld).

[6] BoulON, G., voir référence [5], p. 603.

[7] Auzel, F. et Moncorgé, R., J. Optics 15 (1984) 338.
[8] Spectroscopy of Solid State Laser Type Materials, ed. B. Di Bartolo (Plenum Press, New York, London, ASI Series) 1986. Cours présentés à l'Ecole d'Eté OTAN d'Erice (Sicile) Juin 1985. Parmi les cours intéressant les matériaux inorganiques, mentionnons :

- Recent trends in laser material research (R. Pappalardo).

- Spectroscopy of ionic solid-state laser materials (B. Di Bartolo).

- Spectroscopy of colour centres (B. Henderson).

- New development in transition metal spectroscopy (F. Imbusch).

- Advances in semi-conductor spectroscopy (M. Sturge).

- Progress in rare-earth and actinide spectroscopy (G. Blasse).

- Spectroscopy of post-transition metal ions (G. Boulon).

- Materials for ionic solid state lasers (F. Auzel).

- Glass lasers and solar applications (R. Reisfeld).

- Recent developments in phosphor materials (R. Pappalardo).

[9] KoECHNER, W., Solid State Laser Engineering (Springer-Verlag, Berlin) 1976.

[10] Moncorgé, R., Jacouier, B., Boulon, G., J. Luminescence 12-13 (1976) 467 et 14 (1976) 337.

[11] Rogemond, F., Pedrini, C., Moine, B., Boulon, G., J. Luminescence 33 (1985) 455.

[12] Lurin, C., PARent, C., Le Flem, G. et HagenmulLER, P., J. Phys. Chem. Solids 46 (1985)' 1083.

[13] REISFELD, R., voir référence [8].

[14] ANDrEws, L. J., LEMPICKI, A. and MCCOLlun, B. C., J. Chem. Phys. 74 (1981) 5526.

[15] Reisfeld, R., J. Less Common Met. 93 (1983) 243. 
[16] Kisilev, A. and Reisfeld, R., Chem. Phys. Lett. 115 (1985) 457.

[17] Durville, F., Champagnon, B., Duval, E., BouLON, G., GAUME, F., Wright, A. F. and Fitch, A. N., Phys. Chem. Glasses 25 (1984) 126.

[18] Champagnon, B., Durville, F., Duval, E., BouLON, G., J. Luminescence 31-32 (1984) 345.

[19] Reisfeld, R., KisileV, A., GReEnBERG, E., BuCH, A. and Ish-Shalom, M., Chem. Phys. Lett. 104 (1984) 153 and 105 (1984) 405.

[20] Bouderbala, M., Boulon, G., Reisfeld, R., Buch, A., Ish-Shalom, M., Lejus, A. M., Chem. Phys. Lett. 121 (1985) 535 and June 86.

[21] Durville, F., Champagnon, B., Duval, E., BouLON, G., J. Phys. Chem. Solids 46 (1985) 701.

[22] Butler, K., Fluorescent Lamp Phosphors Technology and Theory (Pennsylvania University Park) 1980.
[23] Ryan, F. M., J. Luminescence 24-25 (1981) 827.

[24] GriffithS, J., J. Ind. Min 199 (1984) 19.

[25] Maestro, P. et Dougier, P. L'actualité Chimique (France) 15 Juin-Juillet 1982.

[26] Saubat, B., Vlasse, M. and Fouassier, C., J. Solid State Chem. 34 (1980) 271.

[27] Saubat, B., Fouassier, C., Hagenmuller, P. and Bourcet, J. C., J. Mater. Res. Bull. 16 (1981) 193.

[28] Photon, Lux 128 (1984) 20.

[29] PAPpalardo, R. G., voir référence [8].

[30] Blasse, G., J. Less Common Metals 112 (1985) 1.

[31] Inokuti, M. and Hirayama, F., J. Chem. Phys. 43 (1965) 1978.

[32] YoкотA, M. and TANimoto, O., J. Phys. Soc. Japan 22 (1967) 779.

[33] McColl, J. R. and Palilla, F. C., ACS Symp. Ser. 164 (1981) 177. 